\title{
The influence of differential evaporation on the structure of a three-component biofuel spray
}

International J of Engine Research 2015, Vol. 16(5) 610-626 (C) IMechE 2015

Reprints and permissions: sagepub.co.uk/journalsPermissions.nav DOI: 10.1 I 77// 468087415573800 jer.sagepub.com

\$SAGE

\author{
Peter Keller', Tobias Knorsch ${ }^{2}$, Michael Wensing ${ }^{2}$ and Christian Hasse'
}

\begin{abstract}
A high-pressure spray injected under gasoline engine conditions in a constant volume chamber is studied in detail using a three-dimensional computational fluid dynamics approach. Both single-component and ternary mixture ( $n$-alkane and alcohols) spray results are compared with the experimental data from shadowgraphy, Schlieren imaging and phaseDoppler anemometry. Activity coefficient models are used to cover non-ideal thermodynamic effects occurring when mixtures of structurally dissimilar components such as alcohols and n-alkanes are considered. Here, the non-random, two-liquid and universal quasi-chemical functional group activity coefficients methods are applied. A ternary mixture composed of ethanol, n-butanol and n-hexane representing the research octane number (RON 95) of gasoline is considered and investigated in detail. To illustrate the non-ideal mixture effects, first the binary mixtures ethanol/ n-butanol, n-butanol/n-hexane and ethanol/n-hexane are studied as subsets of the ternary mixture using a zerodimensional single-droplet evaporation solver. Introducing a differential evaporation factor for multicomponent mixtures, the mixture segregation is characterized. Additionally, a separation factor indicating which component depletes first (volatility) is presented for both binary and ternary mixtures. The zero-dimensional results are then correlated with the three-dimensional computational fluid dynamics simulation results. Looking at the vapor-liquid equilibria of the different binary mixtures, the non-ideal effects are identified. The evaporation behavior of the binary mixtures is compared with the ternary mixtures in terms of droplet lifetimes, temperature and mixture decomposition, where the latter is characterized with the differential evaporation factor and separation factor. These results are afterwards compared with the three-dimensional computational fluid dynamics simulations, which are validated against the experimental data for single-component sprays regarding the liquid and vapor penetration depths. Finally, in the ternary mixture, $\mathrm{n}$-butanol is replaced with iso-octane, the most widely used gasoline surrogate, to illustrate the differential evaporation behavior for a mixture with a higher alkane content than the ternary mixture used for the experiments. Strong non-ideal effects causing significant changes in the vapor composition are presented, as well as changes in the characterization factors, differential evaporation factor and the separation factor, respectively. These results are compared with the single-droplet simulations.
\end{abstract}

\section{Keywords}

Multicomponent fuel evaporation, azeotrope, differential evaporation, separation factor, rapid mixing model

Date received: 28 September 20I4; accepted: 25 January 2015

\section{Introduction}

For modern gasoline engines, a detailed understanding of the spray and vapor formation of multicomponent mixtures is of great importance. Since biofuel mixtures are composed of many different types of hydrocarbons such as alcohols, n- and iso-alkanes or aromatics, the ternary mixture considered in this work contains ethanol, n-butanol and n-hexane (EBH). Because structurally dissimilar components are expected to behave non-ideally and usually form azeotropes, there is a need

\footnotetext{
'Department of Energy Process Engineering and Chemical Engineering, Technische Universität (TU) Bergakademie Freiberg, Freiberg, Germany ${ }^{2}$ Department of Engineering Thermodynamics, Friedrich-AlexanderUniversität Erlangen-Nürnberg, Erlangen, Germany
}

Corresponding author:

Peter Keller, Department of Energy Process Engineering and Chemical Engineering, Technische Universität (TU) Bergakademie Freiberg, Fuchsmuehlenweg 9, Reiche Zeche, Freiberg 09599, Germany. Email: Peter.Keller@iec.tu-freiberg.de 
for a suitable model to describe the vapor-liquid equilibria (VLE), also covering azeotropic behavior. Consequently, the liquid phase behaves non-ideally and hence the focus of this study is to investigate the influence of the activity coefficient models non-random, two-liquid (NRTL) and universal quasi-chemical functional group activity coefficients (UNIFAC) in comparison to the ideal description (Raoult's law).

Numerical simulations can provide an insight into the underlying phenomena which is difficult to obtain by a single experiment alone. However, only consistent thermodynamics describing the relationship between the gas and liquid phase allow the vapor formation to be predicted exactly, which also influences the subsequent combustion. Therefore, a proper model needs to be applied to describe the VLE at the droplet-gas interface, a model that suitably reflects the thermochemical equilibrium between the gas and liquid mixture.

The influence of the chosen VLE description is investigated in detail for the binary mixtures of $\mathrm{EBH}$ as well as for their ternary mixture using a zerodimensional (0D) single-droplet model applying the rapid mixing model (RMM) and a three-dimensional (3D) computational fluid dynamics (CFD) simulation using the Lagrangian approach for the droplet parcels. The multicomponent mixture decomposition induces high species gradients inside the liquid phase, which is why the more sophisticated diffusion limit model (DLM) including one-dimensional species and temperature equations might be the appropriate choice, as demonstrated in Landis and Mills, ${ }^{1}$ Law, ${ }^{2}$ Makino and Law, ${ }^{3}$ Kneer et al., ${ }^{4}$ Sirignano $^{5}$ and Keller et al. ${ }^{6}$ Applying this model, the droplets are resolved in radial layers which have their own temperature and composition. However, because of the high computational effort in 3D CFD simulations, it is not yet feasible. Furthermore, convection effects inside the droplet also affect the species and temperature transport and it is still in discussion ${ }^{5,7,8}$ whether these effects are relevant in real spray applications.

The droplet decomposition is illustrated using a differential evaporation and a separation factor. ${ }^{6,9}$ First, the effect of the chosen VLE on the evaporation behavior of the binary mixtures of the three components is investigated under engine-like conditions for a single droplet, demonstrating the interactions between the different species because of their molecular structure. Furthermore, single-droplet evaporation is simulated using the ideal and non-ideal methods for the ternary mixture with identical conditions. Finally, these results are used to study the effect of thermodynamics in a 3D Lagrangian simulation for high-pressure gasoline spray injection with a six-hole series nozzle.

\section{Numerical model}

The numerical simulations are performed with two different applications. The single-droplet evaporation model is implemented in an in-house code suitable for simulating multicomponent evaporation with different non-ideal thermodynamics. The 3D CFD simulations are performed with OpenFOAM ${ }^{\circledR}$ (version 2.0.x), modified to account for non-ideal multicomponent mixtures. Since both applications use a 0D RMM for the individual droplet, the liquid phase equations are valid for both implementations. However, the gas phase description differs. For single-droplet simulations, it can be assumed to be quasi-steady ${ }^{5,10,11}$ while for the Lagrangian description conservation equations for mass, momentum and energy, as well as species transport equations, have to be solved in $3 \mathrm{D}$.

\section{Gas phase}

Subsequently, the two different descriptions of gas phase treatment are given for the single 0D droplet followed by the 3D model.

Single-droplet model. Only quiescent atmospheres are considered to demonstrate the binary and ternary mixture evaporation of single droplets in section "Results." The species transport and energy conservation equations can be used to derive the gas phase equations. The diffusion equations for the mass fractions of species $i$ in spherical coordinates for a multicomponent mixture are given as ${ }^{12}$

$$
\dot{m}_{F} Y_{i, v}-4 \pi \rho_{g} D_{i m}^{g} r^{2} \frac{d Y_{i, v}}{d r}=\Omega_{i} \dot{m}_{F}
$$

with the overall fuel evaporation rate $\dot{m}_{F}$, the fractional evaporation rate $\Omega_{i}=\dot{m}_{i} / \dot{m}_{F}$, the mass fraction of vapor species $i$ in the gas phase $Y_{i, v}$, the gas phase density $\rho_{g}$, the diffusion coefficient $D_{i m}^{g}$ of species $i$ and the radial coordinate in the gas phase $r$ defined in $r_{s} \leqslant r<\infty$, respectively. The subscript $s$ denotes the surface values and $r_{s}$ is the droplet radius. Similarly, the energy conservation equation can be written according to ${ }^{11-13}$

$$
\dot{m}_{F} c_{p, v}\left(T_{g}-T_{s}\right)+\dot{m}_{F} L+\dot{q}_{l}=4 \pi r^{2} \lambda_{g} \frac{d T_{g}}{d r}
$$

with heat capacity at constant pressure $c_{p}$, the gas and surface temperatures $T_{g}$ and $T_{s}$, the latent heat of vaporization at droplet temperature related to all evaporating species $L=\sum_{i} \Omega_{i} \Delta h_{v, i}$, the heat on the inner droplet surface responsible for droplet heating $\dot{q}_{l}$ and the thermal conductivity $\lambda$, respectively. The gas phase (together with the vapor species, marked by $v$ ) is denoted by the subscript $g$. The heat flux on the inner droplet surface is given by

$$
\dot{q}_{l}=4 \pi \lambda_{g} r_{s}\left(T_{g, \infty}-T_{s}\right) \frac{z}{e^{z}-1}-\dot{m}_{F} L
$$

with 


$$
z=\frac{\dot{m}_{F} c_{p, v}}{4 \pi \lambda_{g} r_{s}}
$$

If $\mathrm{Le}=1$ in the gas phase $\left(\lambda_{g} / c_{p, g}=\rho_{g} D_{i m}^{g}\right)$ and $c_{p, g}=c_{p, v}$ are assumed, the integration of equations (1) and (2) leads to the total mass evaporation rate

$$
\dot{m}_{F}=4 \pi r_{s} \frac{\lambda_{g}}{c_{p, g}} \ln \left(1+B_{m}\right)
$$

with the Spalding mass transfer number $B_{m}$ according to

$$
B_{m}=\frac{Y_{F, s}-Y_{F, \infty}}{1-Y_{F, s}}
$$

Here, $Y_{F, s}=\sum_{i} Y_{i, v}$ denotes the sum of all fuel vapor mass fractions at the droplet surface and $Y_{F, \infty}$ the sum of all fuel vapor mass fractions in the surrounding gas phase infinitely far from the droplet surface (set to 0 for the single-droplet results and taken from the CFD cell in the 3D simulations). A discussion of the potential implications with respect to these assumptions is given in Sirignano ${ }^{5}$ and Sazhin. ${ }^{11}$ To correlate the gas and liquid phases, the vapor mass fractions $Y_{i, v}$ at the interface have to be evaluated using a suitable VLE method discussed in section "VLE."

3D model. The processes have to be described on two scales. The cell size is significantly larger than the droplet diameter where the gas phase equations are solved. The modeled subgrid processes are included in the form of suitable source terms that couple the gas and liquid phases. Since the cases considered are non-reactive, chemical source terms are omitted.

The equations for the species partial densities that are required for multicomponent mixtures read

$$
\frac{\partial \rho_{i}}{\partial t}+\nabla \cdot\left(\rho_{i} \tilde{\mathbf{u}}\right)=\nabla \cdot\left[\bar{\rho} D_{\mathrm{eff}} \nabla \tilde{Y}_{i}\right]+\overline{\dot{\rho}}_{i}^{s}
$$

and

$$
\overline{\dot{\rho}}_{i}^{s}=\frac{\sum_{V} N_{p} \dot{m}_{p, i}}{V}
$$

where $\rho_{i}=\bar{\rho} \tilde{Y}_{i}$ is the partial density of gas species $i, \tilde{Y}_{i}$ the mass fraction of species $i, \tilde{\mathbf{u}}$ the gas velocity field, $D_{\text {eff }}$ the effective diffusivity (including laminar and turbulent diffusion modeling) and $\overline{\dot{\rho}}_{i}^{s}$ the evaporation source term. $N_{p}$ defines the number of droplets inside a Lagrangian parcel. The sum over all species converts equation (7) to the continuity equation

$$
\frac{\partial \bar{\rho}}{\partial t}+\nabla \cdot(\bar{\rho} \tilde{\mathbf{u}})=\overline{\dot{\rho}}^{s}
$$

The gas phase momentum equation is formulated as

$$
\frac{\partial(\bar{\rho} \tilde{\mathbf{u}})}{\partial t}+\nabla \cdot(\bar{\rho} \tilde{\mathbf{u}} \tilde{\mathbf{u}})=-\nabla \bar{p}+\nabla \cdot \sigma+\overline{\mathbf{F}}^{s}+\bar{\rho} \mathbf{g}-\frac{2}{3} \nabla \bar{\rho} \tilde{k}
$$

with the stress tensor

$$
\sigma=\left(\mu+\mu_{t}\right)\left[\nabla \tilde{\mathbf{u}}+(\nabla \tilde{\mathbf{u}})^{T}-2 / 3 \nabla \cdot \tilde{\mathbf{u}} \mathbf{I}\right],
$$

the specific body force $\mathbf{g}$, the kinetic energy $\tilde{k}$ and the spray momentum source term $\overline{\mathbf{F}}^{s} .{ }^{14,15}$ The energy conservation equation in the form of sensible enthalpy reads

$$
\frac{\partial\left(\bar{\rho} \tilde{h}_{s}\right)}{\partial t}+\nabla \cdot\left(\bar{\rho} \tilde{\mathbf{u}} \tilde{h}_{s}\right)=\nabla \cdot\left(\bar{\rho} \alpha_{\mathrm{eff}} \nabla \tilde{h}_{s}\right)+\frac{d \bar{p}}{d t}+\overline{\dot{Q}}^{s}
$$

with the sensible enthalpy $\tilde{h}_{s}$, effective thermal diffusivity $\alpha_{\text {eff }}=\alpha+\mu_{t} /\left(\bar{\rho} \operatorname{Pr}_{h}\right)$ and source term due to heat transferred from and to the parcels and due to evaporation $\bar{Q}^{s}$. The quantities denoted by a tilde are Favre-averaged. The turbulent kinetic energy $\tilde{k}$ and the turbulent dissipation $\tilde{\varepsilon}$ are solved using the $k-\varepsilon$ model by Launder and Sharma. ${ }^{16}$ This model has shown good results in spray injection studies before. ${ }^{17}$

\section{Liquid phase}

In both numerical models, the liquid phase is described using the RMM, which accounts for homogeneous species and temperature distribution inside the droplets. The underlying equations can hence be used for the $0 \mathrm{D}$ and $3 \mathrm{D}$ simulation methods. A detailed discussion has been presented in Keller et al. ${ }^{6}$ and Bader et al. ${ }^{9}$ Only a short summary is given below.

The evaporation rate of species $i$ is described by

$$
\frac{d m_{i}}{d t}=-\dot{m}_{i}=-\Omega_{i} \dot{m}_{F}
$$

The sum over all liquid components yields the total mass change in the droplet

$$
\frac{d m_{d}}{d t}=-\dot{m}_{F}=-\sum_{i} \dot{m}_{i}
$$

The radius and components' mole fractions are given by

$$
\frac{d\left(\rho_{i, l} r_{s}^{3}\right)}{d t}=-\frac{3}{4 \pi} \Omega_{i} \dot{m}_{F}
$$

and

$$
\frac{d\left(X_{i, l} r_{s}^{3} / V_{m, l}\right)}{d t}=-\frac{3}{4 \pi M_{i}} \Omega_{i} \dot{m}_{F}
$$

with the partial liquid density $\rho_{i, l}$, the liquid mole fractions $X_{i, l}$ and the mixture molar volume $V_{m, l}$. The temporal change in the surface temperature is determined by

$$
\frac{d T_{s}}{d t}=\frac{\dot{q}_{l}}{m_{d} c_{p, l}}=\frac{4 \pi r_{s}^{2} \alpha\left(T_{g, \infty}-T_{s}\right)-\dot{m}_{F} L}{\frac{4}{3} \pi r_{s}^{3} \rho_{l} c_{p, l}}
$$

with $\dot{q}_{l}$ by equation (3) and $\dot{m}_{F}$ by equation (5). The heat transfer coefficient $\alpha$ is calculated with 


$$
\alpha=\frac{\dot{m}_{F} c_{p, g}}{4 \pi r_{s}^{2}\left(e^{z}-1\right)}
$$

The Lagrangian droplet description in 3D additionally needs a momentum equation accounting for the droplet movement and coupling to the gas phase

$$
\frac{d \mathbf{u}_{d}}{d t}=-\frac{3 \rho_{g} C_{d}\left(\mathbf{u}_{d}-\tilde{\mathbf{u}}\right)\left|\mathbf{u}_{d}-\tilde{\mathbf{u}}\right|}{4 \rho_{l} d}+\mathbf{g}
$$

where $C_{d}$ denotes the droplet drag coefficient. The droplet diameter at the time step $(n)$ is determined by

$$
d^{(n)}=\sqrt[3]{\frac{6 m_{d}^{(n)}}{\pi N_{p} \rho_{l}}}
$$

noting that in the Lagrangian equations are not solved for droplets but for stochastic parcels containing droplets of the same size, mass, composition and temperature.

\section{VLE}

The vapor mass fractions at the liquid-gas interface $Y_{i, v}$ are determined applying a suitable VLE. In general, using the $\gamma-\varphi$ approach, the VLE can be written as

$$
X_{i, v}=\gamma_{i} X_{i, l} \frac{p_{\mathrm{vap}, \mathrm{i}}}{p} \theta_{i}
$$

which simplifies to Raoult's law, if the activity coefficients $\gamma_{i}$ and the gas phase correction $\theta_{i}$ are unity. The latter combines the gas fugacity coefficients and the Poynting correction. ${ }^{6,9}$ The cases considered in this work operate on moderate pressures up to 8 bar only, which is why $\theta_{i} \approx 1$ and hence gas non-idealities can be neglected. The vapor pressures $p_{\text {vap,i }}$ can be determined by appropriate polynomials such as the Wagner equation. ${ }^{18}$ The vapor mass fractions are obtained from the vapor mole fractions $X_{i, v}$ by

$$
Y_{i, v}=X_{i, v} \frac{M_{i}}{M_{m, g}}
$$

with the mixture molar weight $M_{m, g}=\sum_{i} X_{i, g} M_{i}$. Here, the liquid activity coefficients $\gamma_{i}$ are determined by either the NRTL or the UNIFAC method. Detailed descriptions of these two models are given in Renon and Prausnitz ${ }^{19}$ and Gmehling et al., ${ }^{20}$ respectively.

\section{Experimental and numerical setup}

The 3D CFD simulations are compared with the experiments described and validated for binary biofuel mixtures in Keller et al. ${ }^{21}$ The experimental setup is depicted in detail in Knorsch et al. ${ }^{22}$ In general, the optically accessible spray chamber is operated under gasoline engine conditions to study the liquid and vapor penetration as well as droplet sizes. A detailed overview over the different experimental techniques is

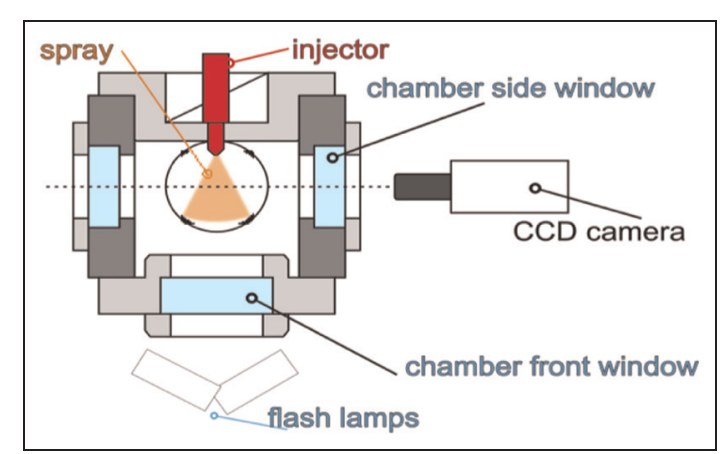

Figure I. General setup of experimental spray chamber according to Knorsch et al. ${ }^{23}$

Table I. Experimentally investigated operating points.

\begin{tabular}{lll}
\hline Operating point & $T_{g}$ & $p_{g}$ \\
\hline$\# 1$ & $473 \mathrm{~K}$ & $0.56 \mathrm{MPa}$ \\
$\# 2$ & $673 \mathrm{~K}$ & $0.8 \mathrm{MPa}$ \\
\hline
\end{tabular}

provided in Keller et al. ${ }^{21}$ and Knorsch et al. ${ }^{23}$ The general setup of the chamber is depicted in Figure 1.

To study different operating points, the ambient temperature and pressure, as well as the rail pressure, can be varied independently. The operating points considered are listed in Table 1. To measure the liquid and vapor penetration and droplet sizes, three optical windows are provided.

The chamber is operated with inert nitrogen atmosphere in all considered cases. The gas flow with velocity between 3 and $10 \mathrm{~cm} / \mathrm{s}$ can be considered quiescent compared to the velocity of the liquid jet around $100 \mathrm{~m} / \mathrm{s}$. The injection pressure is at $20 \mathrm{MPa}$ and the liquid temperature conditioned to $T_{l}=353 \mathrm{~K}$. The injection duration was set to $1 \mathrm{~ms}$ after visual start of injection (avSOI), which also includes electrical activation leading to a delay in the spray injection of about $200 \mu \mathrm{s}$, which is compensated for at the end of the injection.

Several liquid mixtures and single-component sprays have been investigated. The liquid mixture considered in this work is composed of $\mathrm{EBH}(75,15,10 \mathrm{vol} \%)$, which was designed to cover the research octane number (RON) of gasoline (RON 95).

Examples of the experimental results for the liquid and vapor penetration measured with shadowgraphy and Schlieren imaging, respectively, are shown in Figures 2 and 3 for ethanol at $1 \mathrm{~ms}$ avSOI and OP \#1.

\section{Numerical model}

The equations given above were implemented in two different frameworks. The single-droplet solver is written in an in-house program able to calculate the droplet evaporation of multicomponent mixtures. The Eulerian-Lagrangian CFD solution is obtained with 


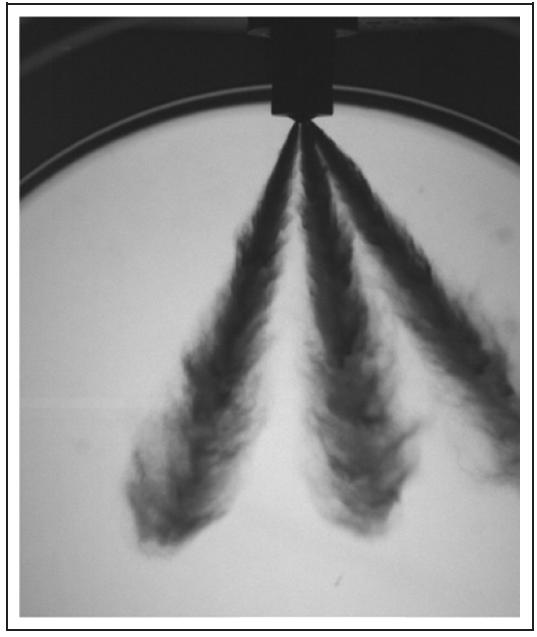

Figure 2. Shadowgraphy image of ethanol spray penetration at I $\mathrm{ms}$ avSOI and OP \#I.

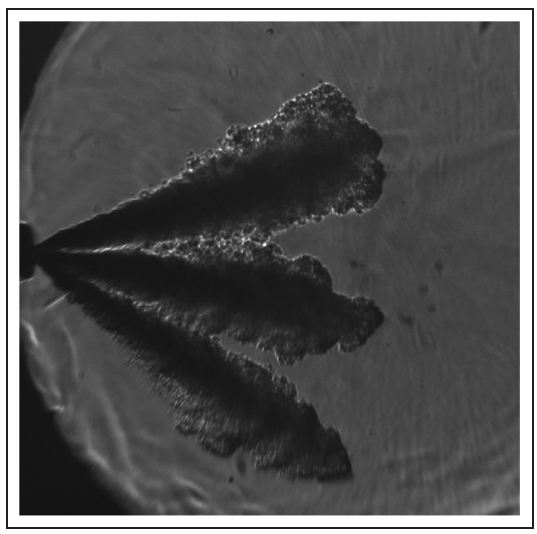

Figure 3. Schlieren image of ethanol vapor formation at I ms avSOI and OP \#I.

the finite volume code OpenFOAM ${ }^{\circledR}$. For the first, a droplet of size $100 \mu \mathrm{m}$ is evaporated at operating conditions equal to those used in the 3D simulation. For the latter, a 3D homogeneous mesh with initially 20,000 cells with a length of $2 \mathrm{~mm}$ is provided, refined adaptively depending on the local fuel-to-gas ratio ${ }^{24}$ up to 400,000 cells, which yields a length of $0.5 \mathrm{~mm}$ for a two-level refinement. There are no breakup or atomization models considered here. The initial droplet sizes are prescribed by a Rosin-Rammler distribution adapted to phase-Doppler anemometry (PDA) measurement data given at an axial distance of $15 \mathrm{~mm}$. The considered mixtures are given in Table 2, where the binary mixtures are composed in the ratio the components are contained in the ternary mixture EBH.

\section{Results}

Subsequently, the single-droplet and spray results are discussed for single components, binary and ternary mixtures of EBH. The main aspect of the investigations is to work out the influence of non-ideal thermodynamics on the evaporation and the mixture segregation/differential evaporation.

\section{VLE modeling}

The isobaric VLE of a binary mixture is characterized by two curves representing the boiling and condensation temperatures for all possible mole fractions. The curves are limited by the pure component states, which are the boiling temperatures. For each temperature between these two limits, an isothermal line provides the corresponding liquid and vapor composition. Inversely, for a given liquid composition, the boiling temperature can be found vertically from the mole fraction value and the vapor composition horizontally to the intersection point.

Figure 4 represents the VLE of ethanol/n-butanol mixtures at $p=1.013$ bar. It can be seen that the ideal and non-ideal methods (NRTL and UNIFAC) correlate very well with the experimental data from Seo et al. ${ }^{25}$ For binary mixtures of these two alcohols with n-hexane (n-alkane), the behavior is completely different. The VLE of ethanol/n-hexane and n-butanol/ n-hexane mixtures are depicted in Figures 5 and 6, respectively, at $p=1.013$ bar. The results obtained with the ideal method now strongly differ from the experimental data given in Kudryavtseva and Susarev ${ }^{26}$ and Dominguez et al. ${ }^{27}$ Furthermore, the prediction of the boiling and condensation temperatures compared with the experimental data is better with the UNIFAC method than with the NRTL model. These differences occur because the standard model coefficients were applied without adaption to the experimental data. Hence, the UNIFAC method provides better results with the standard coefficients and is therefore more suitable for the cases considered here. For other mixtures, as e.g. discussed in Bader et al. ${ }^{9}$ and Keller et al., ${ }^{21}$ the NRTL model may give comparable or even better results than the UNIFAC method. Additionally, it can be seen that the alcohols form azeotropes with nhexane. Depending on the mixture composition, which can be either to the left or right of the azeotropic point, the evaporation behavior of the mixture changes and the highly volatile component (based on the vapor pressure) might remain inside the droplet, as discussed in detail in Keller et al. ${ }^{6}$ Because the UNIFAC method has shown a better agreement with the experiments for the considered mixtures, it will be used for the investigations discussed below.

\section{Single-droplet model}

In the previous section, the VLE of the binary mixtures of the three combinations of EBH was investigated to characterize a ternary mixture $(\mathrm{EBH})$ of these three components. The results are now assigned to singledroplet investigations, where the ratios of the ternary mixture components are used to initialize the binary mixtures. The setup is given in Table 2. The reason for 
Table 2. Single-component fuels, binary and ternary mixtures investigated and their composition.

\begin{tabular}{|c|c|c|c|}
\hline \multicolumn{4}{|l|}{ Single components } \\
\hline Components & Ethanol & n-Butanol & \\
\hline Volume fractions & 1.0 & 1.0 & \\
\hline \multicolumn{4}{|l|}{ Binary mixtures } \\
\hline Components & Ethanol/n-butanol & Ethanol/n-hexane & n-Butanol/n-hexane \\
\hline Volume fractions & $0.83 / 0.17$ & $0.88 / 0.12$ & $0.6 / 0.4$ \\
\hline \multicolumn{4}{|l|}{ Ternary mixtures } \\
\hline Components & $\mathrm{EBH}$ & EIH & \\
\hline Volume fractions & $0.75 / 0.15 / 0.1$ & $0.75 / 0.15 / 0.1$ & \\
\hline
\end{tabular}

EBH: ethanol/n-butanol/n-hexane; ElH: ethanol/iso-octane/n-hexane.

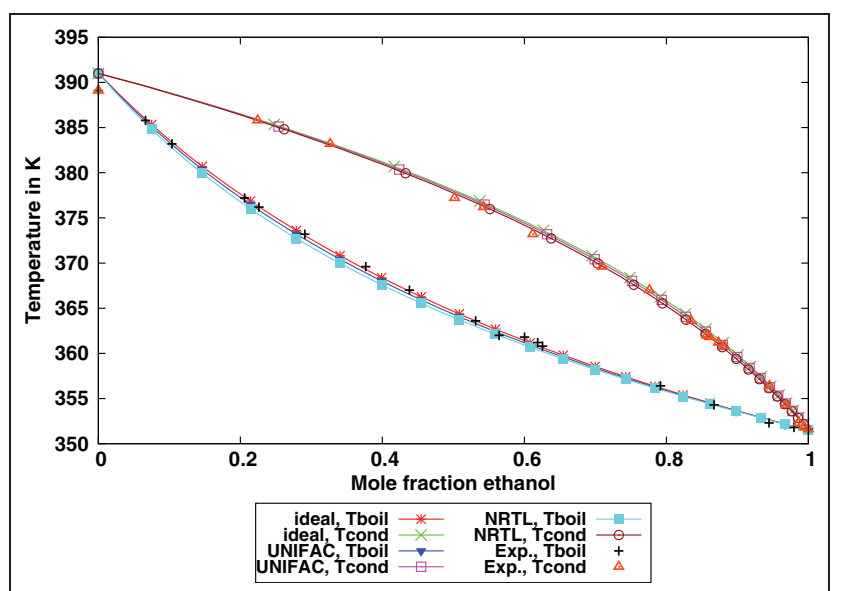

Figure 4. Calculated VLE of ethanol/n-butanol mixtures compared with experimental data from Seo et al..$^{25}$ at $p=1.013$ bar.

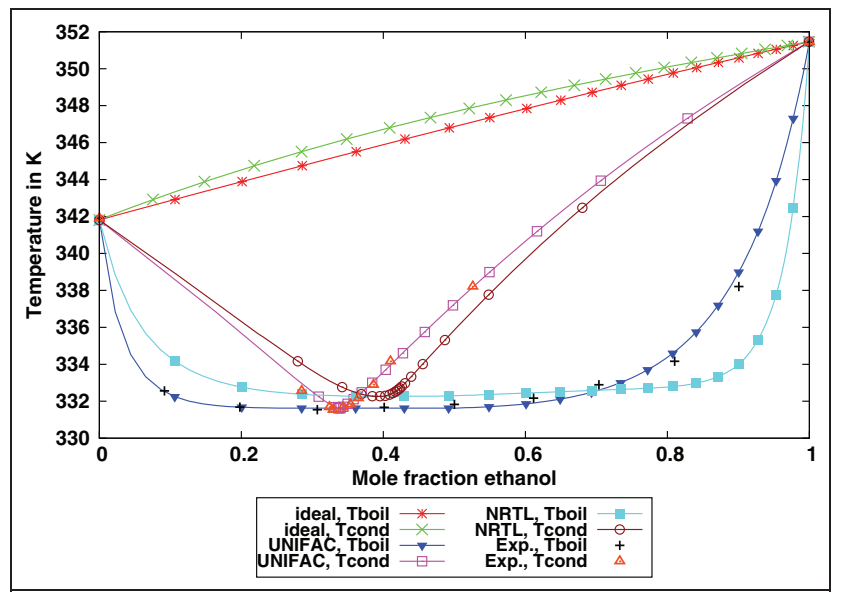

Figure 5. Calculated VLE of ethanol/n-hexane mixtures compared with experimental data from Kudryavtseva and Susarev $^{26}$ at $p=1.013$ bar.

this approach is that differential evaporation effects are much easier to identify for binary than for ternary mixtures. The evaporation progress is investigated by analyzing the decrease in $d^{2}$, where $d$ is the instantaneous diameter. The $d^{2}$ development of the three binary mixtures as well as those of the EBH mixture is given in Figure 7 for $\mathrm{OP} \# 2$. As can be seen, the droplet lifetimes

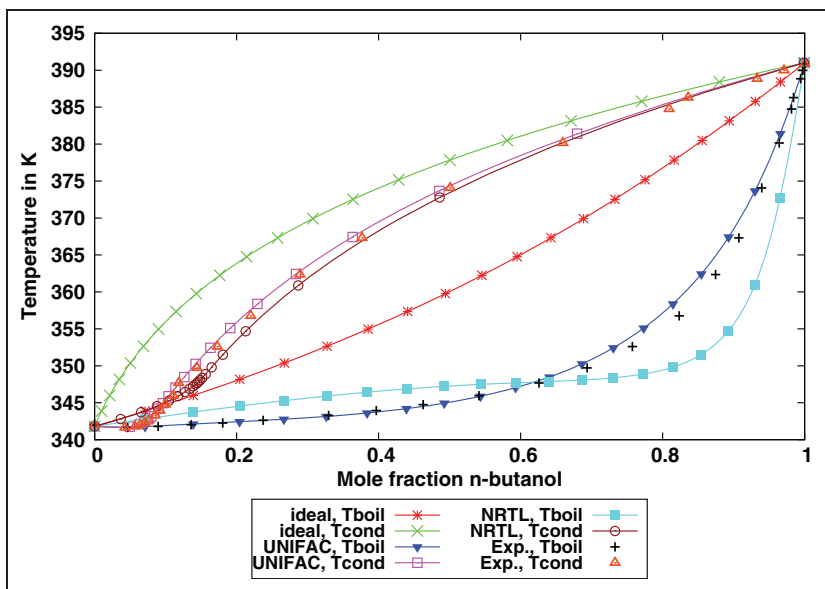

Figure 6. Calculated VLE of $n$-butanol/n-hexane mixtures compared with experimental data from Dominguez et al. ${ }^{27}$ at $p=1.013$ bar.

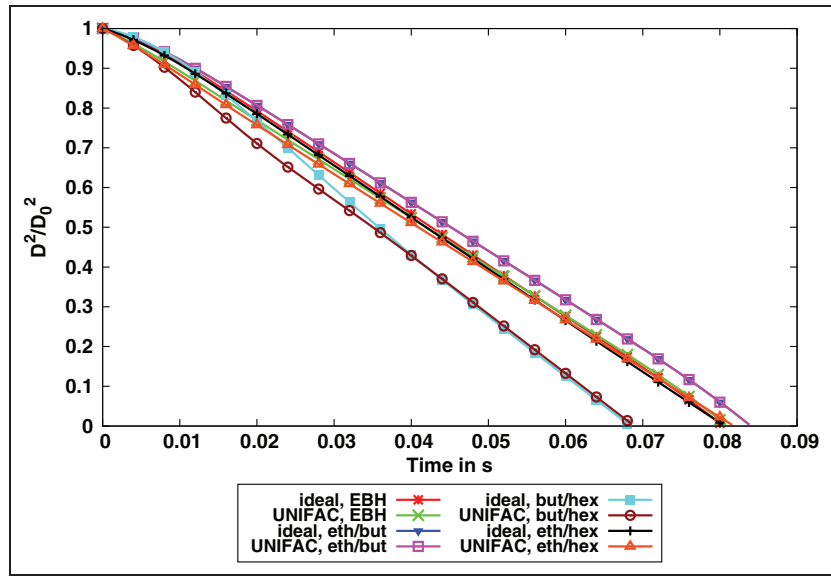

Figure 7. Squared diameter of binary and ternary mixtures simulated using ideal/UNIFAC method.

(DLTs) are not influenced by the thermodynamic model choice. However, the mixtures differ in their evaporation behavior. Because of the high content of $n$ hexane in the n-butanol/n-hexane mixture and its low boiling temperature (and therefore high vapor pressure), this mixture evaporates faster than the others. The ethanol/n-butanol mixture evaporates slowest 


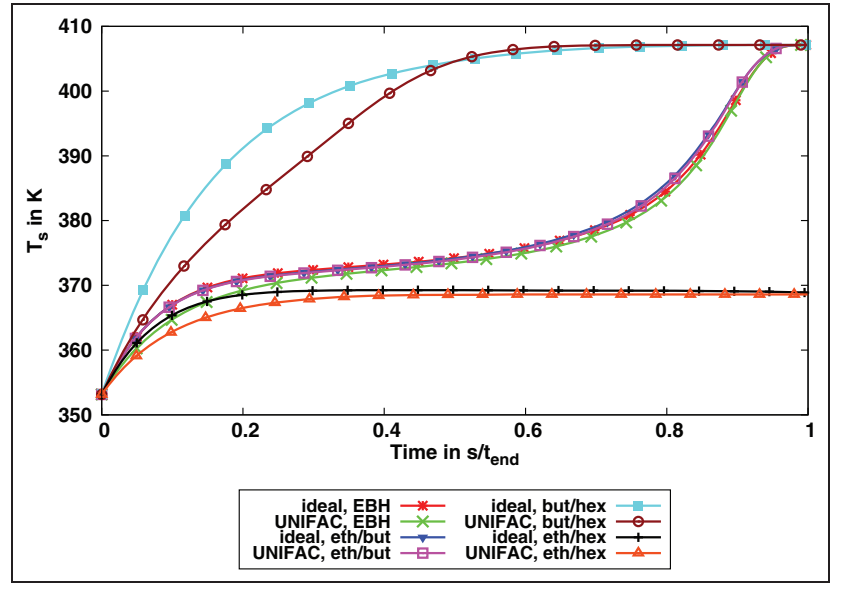

Figure 8. Surface temperatures of binary and ternary mixtures simulated using ideal/UNIFAC method.

because of the high heat of vaporization of ethanol and the high boiling temperature of n-butanol. The values of the heat of vaporization and vapor pressures as a function of temperature are given in Figures 35 and 36 in Appendices 1 and 2.

The temporal evolution (scaled by the end of DLT) of the mixtures' surface temperatures as illustrated in Figure 8 confirms these correlations. The temperature of the binary ethanol/n-hexane mixture increases to a lower level than mixtures containing n-butanol, which always remains as least volatile component inside the droplet as illustrated for the EBH mixture in Figure 9. The n-butanol/n-hexane mixture rapidly increases to a constant temperature that is also reached by the ethanol/n-butanol and EBH mixtures almost at the end of DLT. Since the content of $n$-hexane inside the EBH mixture is very low, the latter two mixtures evaporate almost identically. Only the non-ideal model causes slight deviations from the other results. Nonetheless, in comparison to the EBH mixture, the effect of mixing alcohols and n-alkanes is much higher for the binary cases.

To indicate which component leaves the droplet first, the separation factor can be used. ${ }^{9}$ With the equilibrium constant reading

$$
K_{i}=\frac{X_{i, v}}{X_{i, l}}=\gamma_{i} \frac{p_{\text {vap }, \mathrm{i}}}{p} \theta_{i}
$$

defining the ratio between the vapor and liquid composition, the binary separation factor $\alpha_{i j}$ can be written according to

$$
\alpha_{i j}=\frac{K_{i}}{K_{j}}=\frac{\gamma_{i} p_{\mathrm{vap}, \mathrm{i}} \theta_{i}}{\gamma_{j} p_{\mathrm{vap}, \mathrm{j}} \theta_{j}}
$$

If gas phase non-idealities are neglected, the liquid fugacity (rather than the vapor pressure, as in the ideal case) defines the volatility of the components. Hence, the higher the volatility, the larger the corresponding $K_{i}$. From equation (23), it follows that if component $i$ is

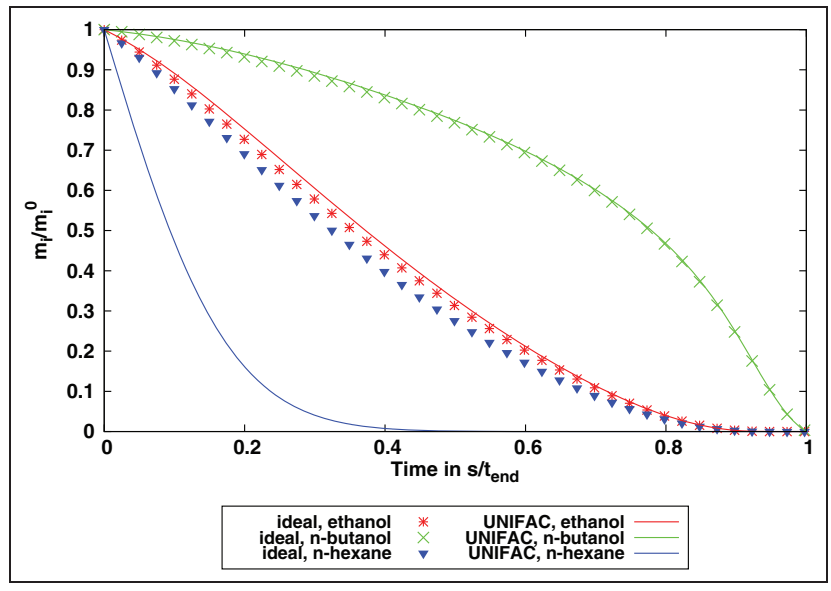

Figure 9. Temporal change in the masses of ternary mixture components at OP\#2.

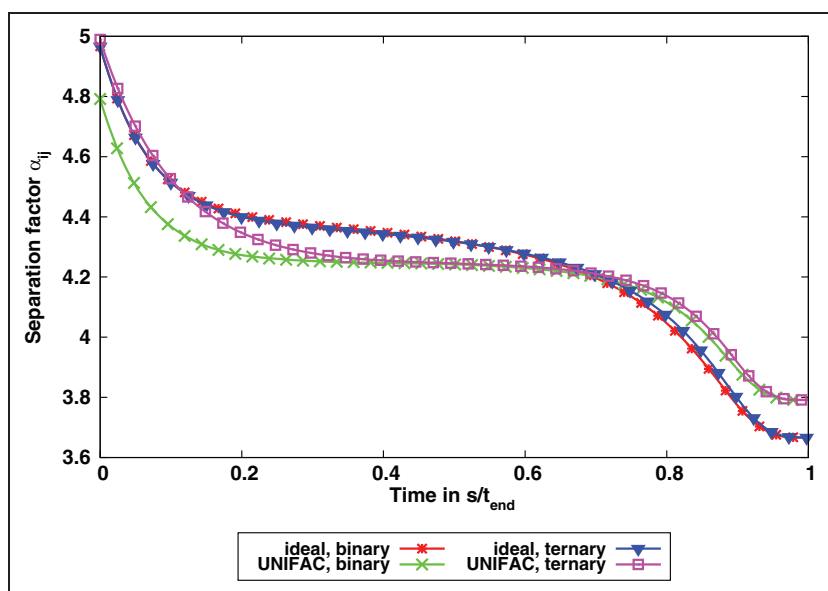

Figure 10. Temporal evolution of separation factors $\alpha_{\text {eth,but }}$ of binary ethanol/n-butanol and ternary EBH mixture at OP\#2.

the more volatile component, $\alpha_{i j}>1$. For the ideal mixtures of the components considered here, in particular, this is always valid, since the polynomials of vapor pressure do not coincide. With activity coefficients obtained with the non-ideal approach, this does not necessarily hold, since they are strongly dependent on the liquid composition. This has been discussed in Bader et al. ${ }^{9}$ The temporal change in the separation factors of the binary mixtures compared with the EBH mixture is presented in Figures 10-12. The factor $\alpha_{\text {eth,but }}$ is almost identical for both mixtures and models. This is because ethanol and n-butanol are the main components inside the EBH mixture considered (90 vol\%). Furthermore, they have a similar structure due to the polar hydroxyl group, which is why the ideal method could be applied at least for the binary mixture of these two components. In the mixture with n-hexane, n-butanol is always the least volatile component and the separation factor $\alpha_{\text {but,hex }}$ is smaller than 1 . Although the value is even lower when the UNIFAC method is applied, this does not change the general 


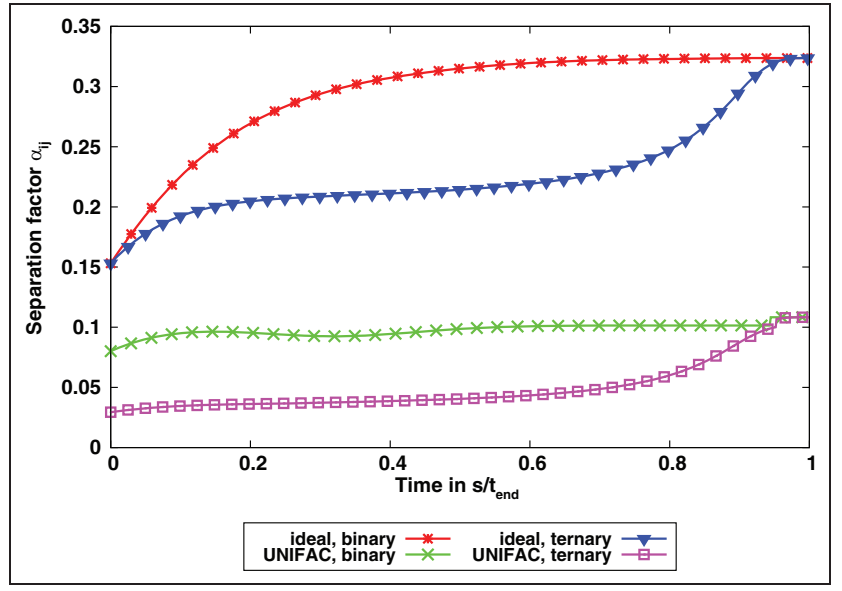

Figure I I. Temporal evolution of separation factors $\alpha_{\text {but,hex }}$ of binary $n$-butanol/n-hexane and ternary EBH mixture at OP\#2.

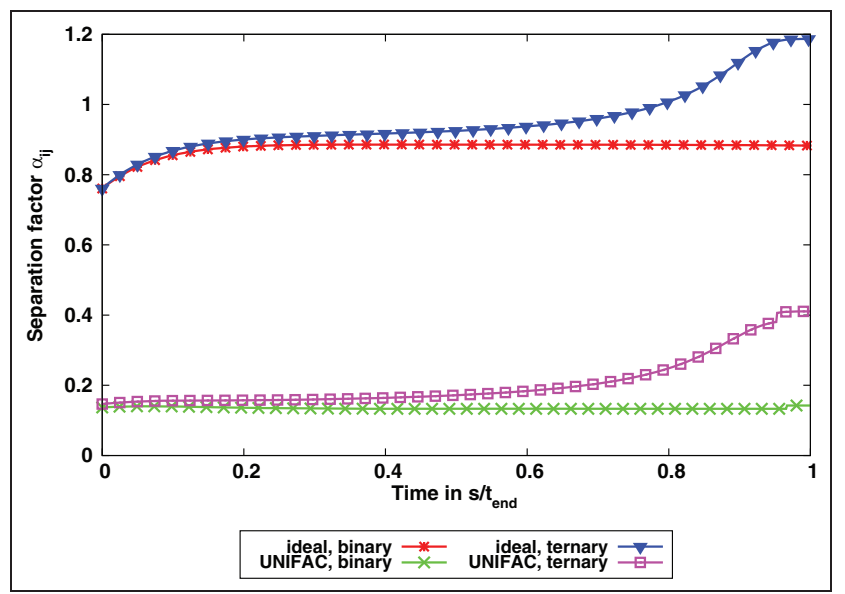

Figure I2. Temporal evolution of separation factors $\alpha_{\text {eth,hex }}$ of binary ethanol/n-hexane and ternary EBH mixture at OP\#2.

evaporation behavior. Finally, the binary ethanol/nhexane mixture shows how applying the non-ideal model influences the separation of components. While, with the ideal model, the components ethanol and nhexane evaporate almost simultaneously for both the binary and ternary mixture, in the case of UNIFAC, nhexane is depleted much faster (see Figure 9 for comparison).

While the separation factor illustrates the instantaneous state of the mixture, the entire evaporation process can be tracked by integrating over the fractional evaporation rate. The differential evaporation factor $(\mathrm{DEF})^{6}$ was introduced for binary mixtures and describes the tendency of the mixture to evaporate in a simultaneous or segregated manner. Because of its symmetry, only one factor is needed to describe the binary mixture segregation

$$
\mathrm{w}_{\mathrm{bin}}=\frac{1}{2 Y_{1, l}^{0}\left(1-Y_{1, l}^{0}\right) m_{0}} \int_{0}^{\Delta t_{d}}\left|\Omega_{1}-Y_{1, l}^{0}\right| \dot{m}_{F} d t
$$

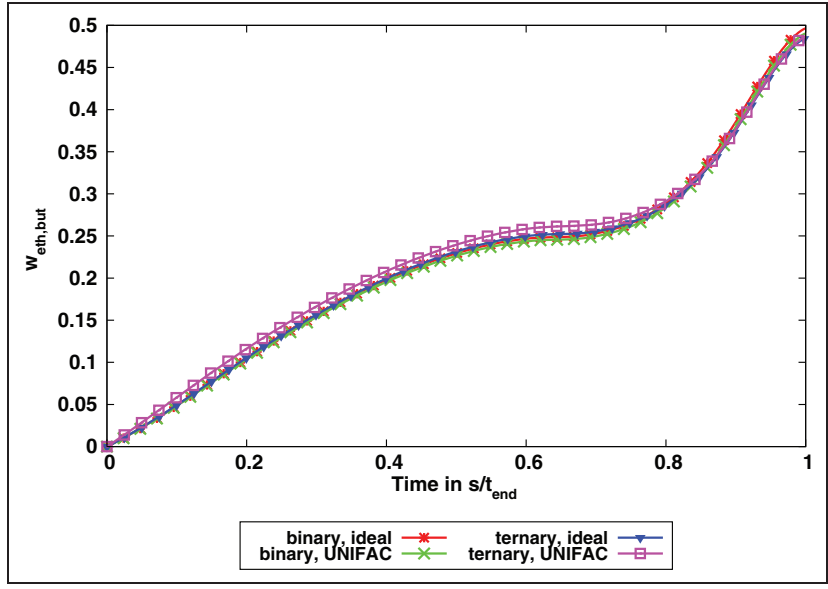

Figure 13. Temporal evolution of DEF of the binary ethanol/nbutanol and the $\mathrm{EBH}$ mixture at OP\#2.

where $Y_{1, l}$ can be chosen arbitrarily from the two components. If $\mathrm{w}_{\text {bin }}=0$, the components would leave the droplet simultaneously; for $\mathrm{w}_{\text {bin }}=1$, they would evaporate in a completely segregated manner. In order to account for multicomponent mixtures, equation (24) has to be slightly modified, since $\mathrm{w}_{\text {bin }}$ is no longer symmetrical. The multicomponent mixture is split into binary ones, where the DEF again describes the evaporation behavior of the corresponding two components. For that reason, two scaled variables representing the current vapor mass fractions $\Theta_{i j}$ and initial liquid mass fractions $\Gamma_{i j}^{0}$ within the binary subset of the multicomponent mixtures are introduced

$$
\begin{aligned}
\Theta_{i j} & =\frac{Y_{i, v}}{Y_{i, v}+Y_{j, v}} \\
\Gamma_{i j}^{0} & =\frac{Y_{i, l}^{0}}{Y_{i, l}^{0}+Y_{j, l}^{0}}
\end{aligned}
$$

The new form of DEF $\mathrm{w}_{i j}$ then reads

$$
\begin{aligned}
\mathrm{w}_{i j} & =\frac{1}{2 \Gamma_{i j}^{0}\left(1-\Gamma_{i j}^{0}\right) m_{0}} \int_{0}^{\Delta t_{d}}\left|\Theta_{i j}-\Gamma_{i j}^{0}\right| \dot{m}_{F} d t \\
& =\frac{Y_{i, l}^{0}+Y_{j, l}^{0}}{2 Y_{i, l}^{0} Y_{j, l}^{0} m_{0}} \int_{0}^{\Delta t_{d}}\left|\Theta_{i j}-\Gamma_{i j}^{0}\right| \dot{m}_{F} d t
\end{aligned}
$$

Again, this factor is symmetric for the components $i$ and $j$. It gives information about how the two components evaporate. The limits of $\mathrm{w}_{i j}$ at 0 and 1 define the rapid regression and sequential evaporation limits. However, it does not provide any information on the general evaporation behavior of the multicomponent mixture, since the third component might behave completely differently. This can also be observed for the considered ternary mixture. In Figures 13-15, the results for the three binary mixtures are compared with the ternary mixture DEFs. Omitting n-hexane does not affect the value of the DEF between ethanol and n- 


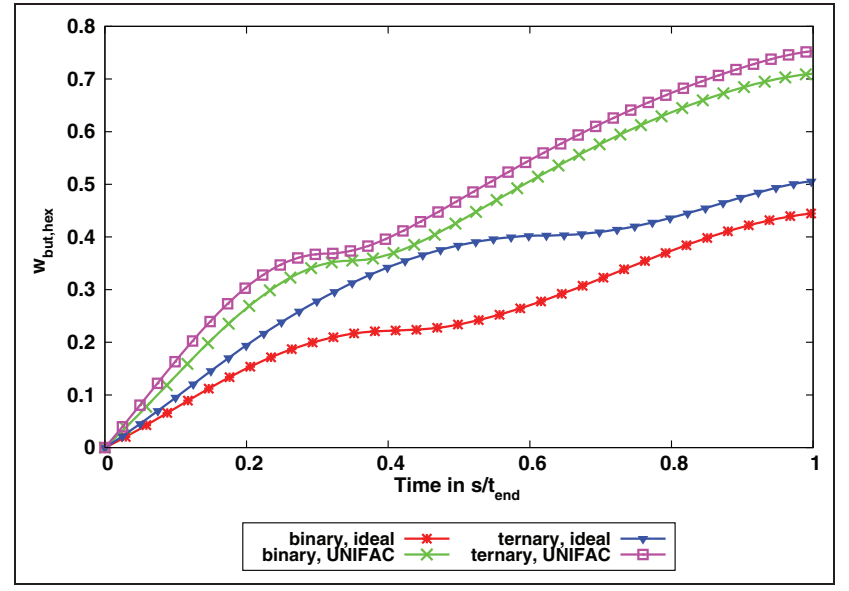

Figure 14. Temporal evolution of DEF of the binary n-butanol/ n-hexane and the EBH mixture at OP\#2.

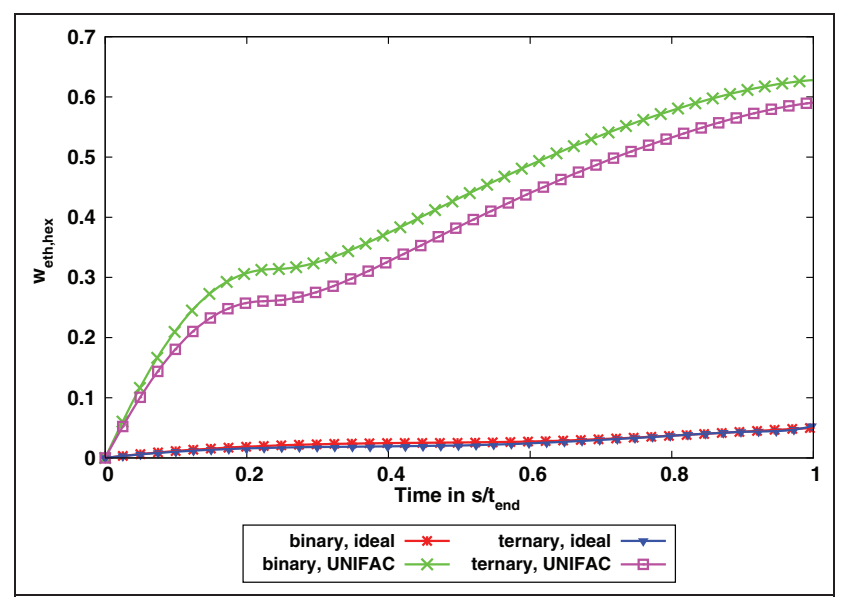

Figure I5. Temporal evolution of DEF of the binary ethanol/nhexane and the $\mathrm{EBH}$ mixture at OP\#2.

butanol. This means that independently of the thermodynamic model used or the content of n-hexane, ethanol and n-butanol will always evaporate with a significant degree of separation. For the mixture of nbutanol with $n$-hexane, the degree of separation is decreased if the ideal model is applied; for the ethanol/ n-hexane mixture, it predicts almost simultaneous evaporation. This conforms the results for the separation factor presented before, where $\alpha$ was almost 1 for ethanol to n-hexane equilibrium constants. The illustration of the components' total mass change inside the droplet further verifies these results (Figure 9). Obviously, the ethanol and n-butanol masses (related to their initial masses) only change slightly when the thermodynamic models are switched. However, the n-hexane mass inside the droplet changes significantly. While it decreases similarly to the mass of ethanol using the ideal approach, it is depleted much earlier for UNIFAC. This indicates that only $\mathrm{n}$-hexane causes strong deviations from ideal behavior, which is due to the molecular interactions between the n-alkane with the two alcohols. However, the results of the binary mixtures and the EBH mixture are very similar.

From the single-droplet investigations above, it can be concluded that although the n-alkane content is very low inside the mixture, the composition and thus the vapor concentration at the surface are affected significantly by the interactions with the alcohols.

Furthermore, the use of DEF and the separation factor also allows the evaporation behavior of multicomponent mixtures to be characterized. Looking at the partial binary mixtures, the mixture segregation can be quantified. In the next section, the multicomponent evaporation and the corresponding spray structure for full 3D simulations are discussed.

\section{Eulerian-Lagrangian CFD simulation}

First, the simulation results of single-component ethanol and n-butanol sprays for $\mathrm{OP} \# 2$ are presented and compared with the experimental data. Following that, the multicomponent spray structure based on the components discussed is investigated applying the UNIFAC model. The last part investigates another ternary mixture, where n-butanol has been replaced by iso-octane, which is the most widely used gasoline substitute in numerical studies.

Single-component sprays. Since vapor penetration measurements are not available for the ternary mixture considered, the simulations have been validated with the single-component experimental data. The liquid penetration depths are given in Figure 16 and the vapor penetration depths in Figure 17. The threshold was set to $1 \%$ of the liquid mass and $0.1 \%$ of the vapor mass fraction for the simulation results and the experimental threshold for the shadowgraphy images at 45 out of 256 grayscale values. For the Schlieren image evaluation, similar values were used. With these thresholds and a droplet size distribution adapted to the ternary mixture's PDA measurement data (section "Ternary mixture simulations"), very good agreement between the two data sets was achieved.

Because multiple injections were recorded (10 for each time step of Schlieren imaging and 32 for shadowgraphy) to cover fluctuations in the spray injection, the minimum and maximum values of the image evaluation are plotted for comparison with the simulation results. Since unsteady Reynolds-averaged Navier-Stokes (URANS) simulations were performed here, only average values for the liquid and vapor penetration are obtained. Although the substance properties such as boiling temperature and vapor pressure, which are relevant for the evaporation characteristics, are very different for the two alcohols, the results are almost identical. During the injection of the spray, the momentum transfer from the liquid to the gas phase is dominant, that is very similar for both species. Furthermore, the threshold for the vapor is very low; hence, only the 


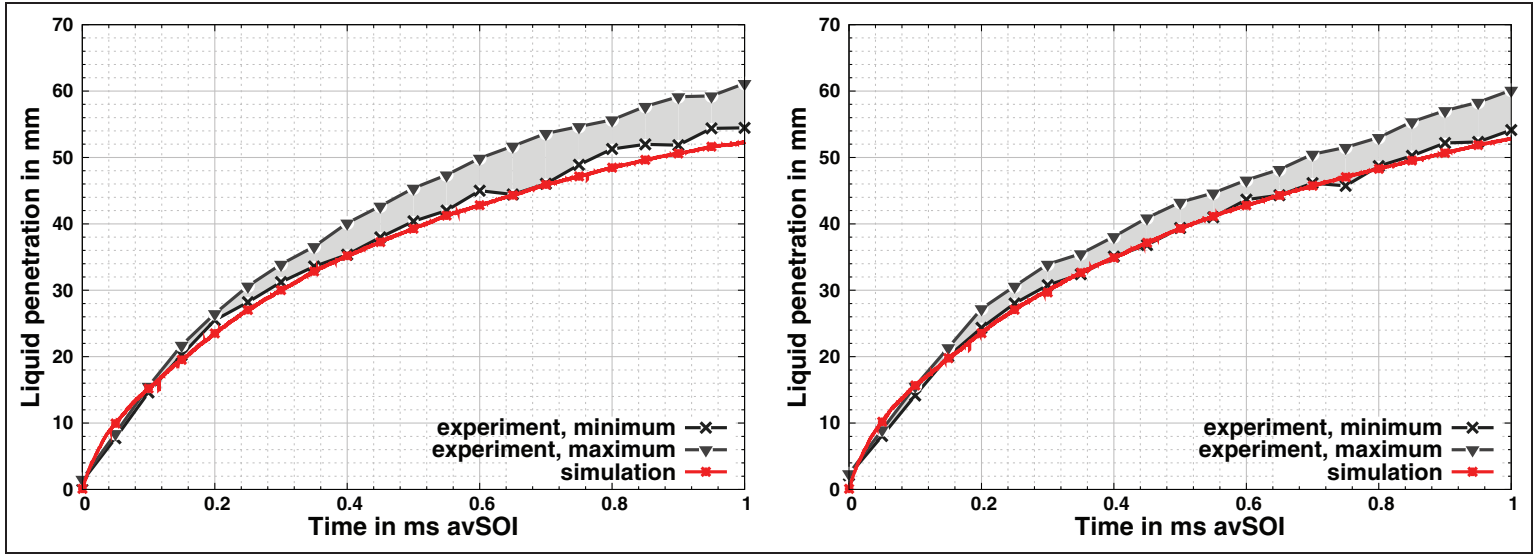

Figure 16. Liquid penetration depths of single-component n-butanol (left) and ethanol (right) sprays at OP\#2.
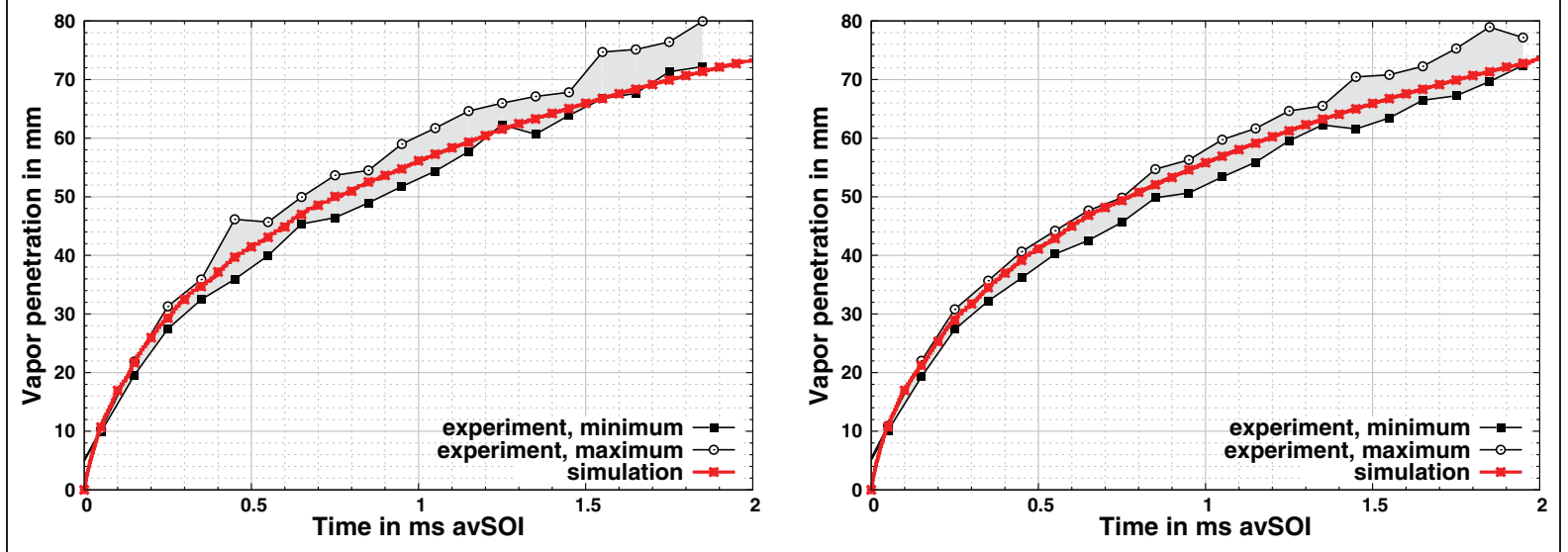

Figure 17. Vapor penetration depths of single-component n-butanol (left) and ethanol (right) sprays at OP\#2.

vapor's occurrence is illustrated, but not its quantification. This can be extended to the ternary mixture, which is mainly composed of these two components and hence gives very similar results.

Ternary mixture simulations. Because of the availability of the experimental data, the initial droplet sizes are provided by a Rosin-Rammler distribution adapted to PDA measurement data. For OP\#1 and \#2, Figures 18 and 19 illustrate the droplet size distribution obtained using PDA measurement, the fitted Rosin-Rammler distribution and the simulation results obtained with the ideal and non-ideal models at 0.5 and $1 \mathrm{~ms}$ avSOI. The experimental data were taken at an axial distance of $15 \mathrm{~mm}$, which is sufficiently far away from the nozzle as discussed in Keller et al. ${ }^{21}$ and Xiao et al. ${ }^{28}$ These data represent the droplet size distribution averaged over five radial positions at the corresponding plane and were sampled over the complete injection time.

Although the droplet size distribution is used at the injection position, the profile is almost exactly recovered at the $15 \mathrm{~mm}$ position, and thus the simulation yields good agreement with the experimental data for both operating points and instants in time.

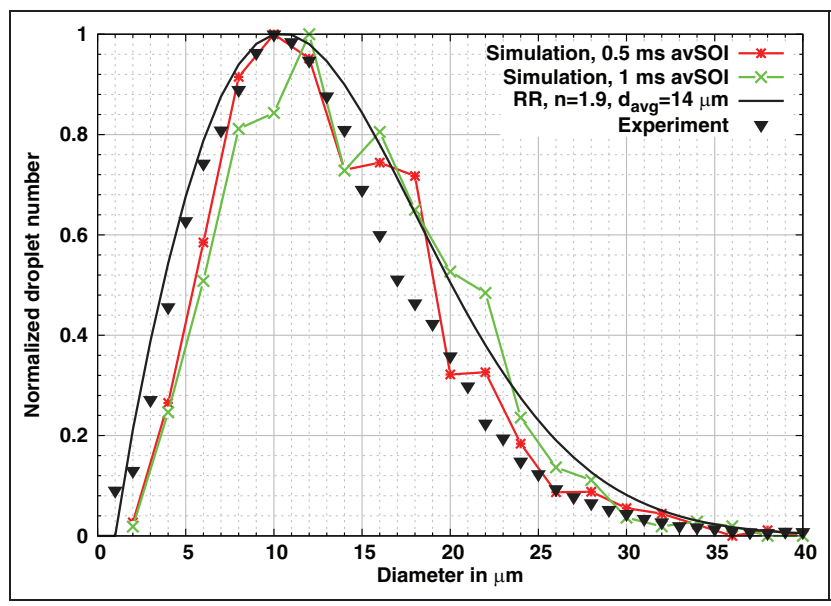

Figure I 8. Droplet size distribution of the EBH mixture determined using PDA data, Rosin-Rammler distribution and the UNIFAC method at $15 \mathrm{~mm}$ axial distance for OP\#I at 0.5 and I ms avSOI.

In Figures 20 and 21, the liquid penetration of the ternary mixture is depicted for OP\#1 and OP\#2, respectively. For the colder case (\#1), the agreement is again very good. There is no unique choice of the threshold 


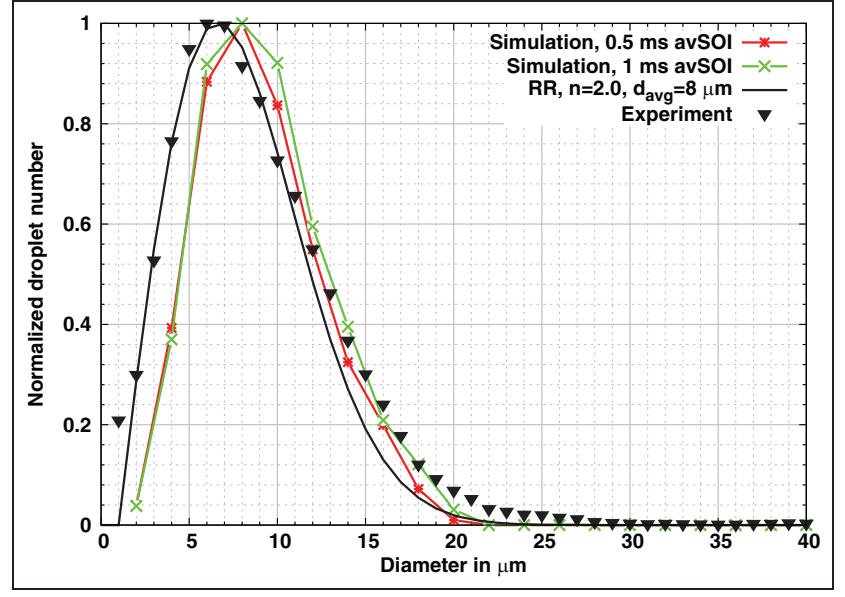

Figure 19. Droplet size distribution of the EBH mixture determined using PDA data, Rosin-Rammler distribution and the UNIFAC method at $15 \mathrm{~mm}$ axial distance for OP\#2 at 0.5 and I ms avSOI.

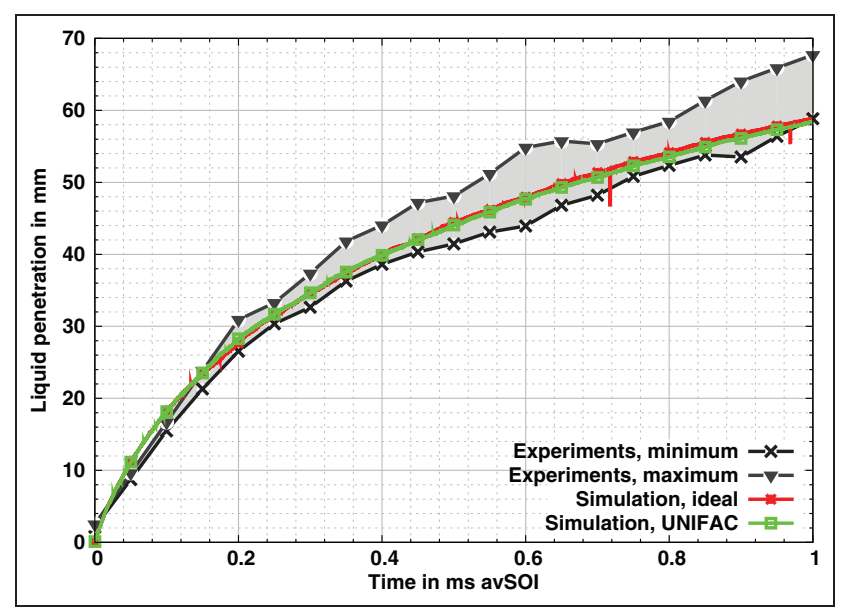

Figure 20. Liquid penetration depths of EBH mixture for OP\#I.

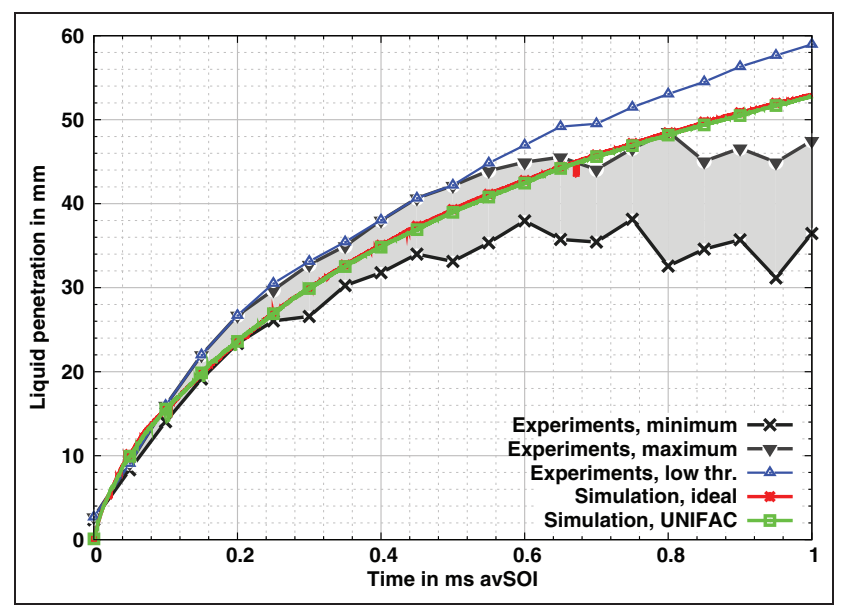

Figure $2 \mathrm{I}$. Liquid penetration depths of $\mathrm{EBH}$ mixture for $\mathrm{OP} \# 2$. for evaluating the shadowgraphy images. Thus, for OP\#2, we also plot a second curve obtained using a lower threshold, showing the sensitivity to this choice especially when comparing to the numerical data, which does predict the steady increase in the penetration depth. Obviously, $99 \%$ of liquid mass seem to be spread over a larger distance in the simulation, which cannot be captured by a fixed threshold for each case. When the threshold is adapted to the experimental data, good agreement with the simulations is found again.

As discussed above, the DLTs do not change if different VLE approaches are used. This is directly reflected in the liquid penetration depth, explaining why no significant difference can be seen. However, the local vapor composition is changed. Looking at Figure 22, large deviations can be found between the two approaches. The left contour plot illustrates the ethanol, the middle plot the n-butanol and the right plot the n-hexane vapor contour profiles. Each of the figures shows the ideal and UNIFAC results. For the components ethanol and n-butanol, the local differences are at first sight very small, and n-hexane indeed strongly differs. This has already been observed before and is fully consistent with the single-droplet studies.

Subsequently, the differential evaporation behavior of the EBH mixture components is analyzed in more detail. After the droplets are evaporated completely, the components' masses must be fully found in the gas phase. Hence, the vapor mass ratio provides information about the state of differential evaporation. For these vapor ratios $Y_{i, v} / Y_{j, v}$ of the components, large differences occur. In Figures 23-25, the vapor mass ratios of the components are shown along the spray axis for $\mathrm{OP} \# 2$, compared with the initial liquid mass ratio. As the contour plots demonstrated, the ethanol-to-n-butanol ratio is very similar for both VLE methods. Because of the initially high evaporation rate of ethanol, the ratio is much higher than at an axial distance of $60 \mathrm{~mm}$, where n-butanol and thus the droplets are completely evaporated. In that situation, the vapor composition is equal to the initial liquid composition. However, for the ratios of ethanol to n-hexane and nbutanol to $n$-hexane, the behavior changes. While the ideal model again predicts the vapor composition as being equal to the initial liquid composition, the vapor mass fractions determined with UNIFAC change. As shown for the single-droplet model in Figure 9, the nhexane evaporation rate is much higher at the beginning and it depletes much faster than with the ideal method, which is why the ratios of ethanol and nbutanol to n-hexane are much lower than the initial liquid ratio. At a distance of $60 \mathrm{~mm}$, the vapor that evaporated earlier has spread much further because of diffusion and the n-hexane mass fraction is much lower than it was simulated with the ideal model. This is very important since the local composition of vapor affects subsequent phenomena such as combustion.

This strong differential evaporation behavior can again be confirmed by the separation and DEFs 


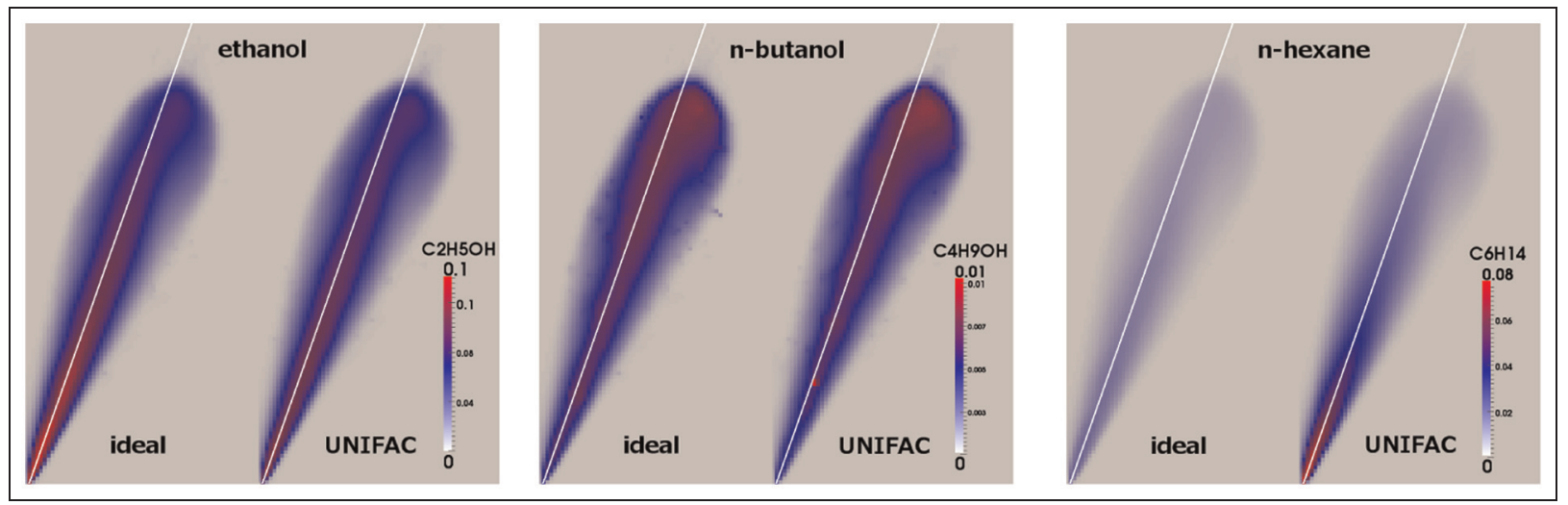

Figure 22. Contour plot of ethanol (left), n-butanol (middle) and n-hexane (right) for the ternary mixture at OP\#2 simulated with ideal (left sprays) and UNIFAC method (right sprays) at I ms avSOI.

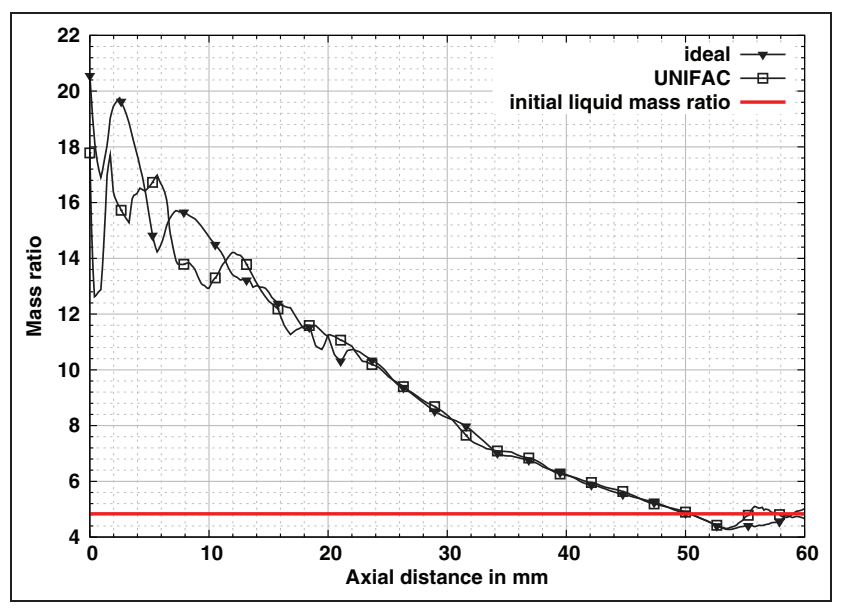

Figure 23. Vapor mass ratio of ethanol to n-butanol along the spray axis for OP\#2 at I ms avSOI.

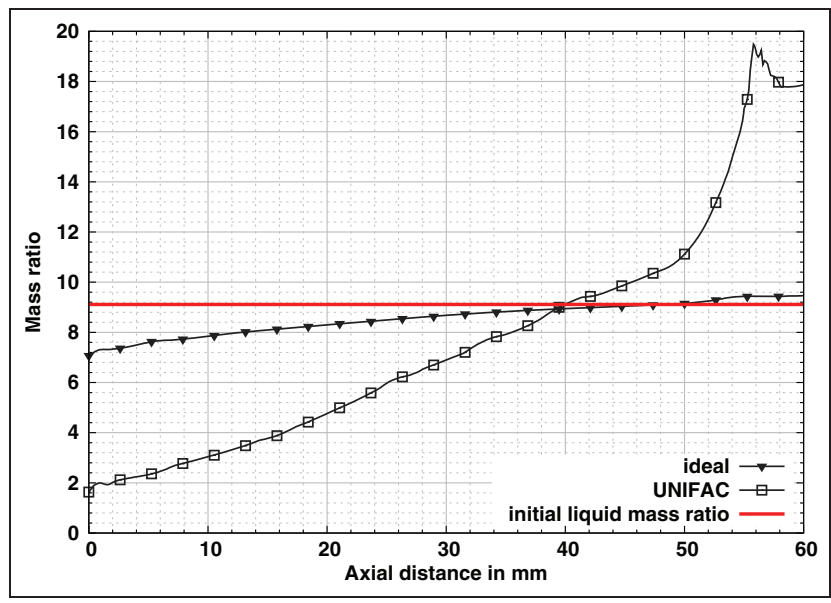

Figure 24. Vapor mass ratio of ethanol to n-hexane along the spray axis for OP\#2 at I ms avSOI.

introduced before, and this is illustrated in Figures 26 and 27 for ethanol and n-hexane at OP\#2 initially and at $1 \mathrm{~ms}$ avSOI, where the normalized number density

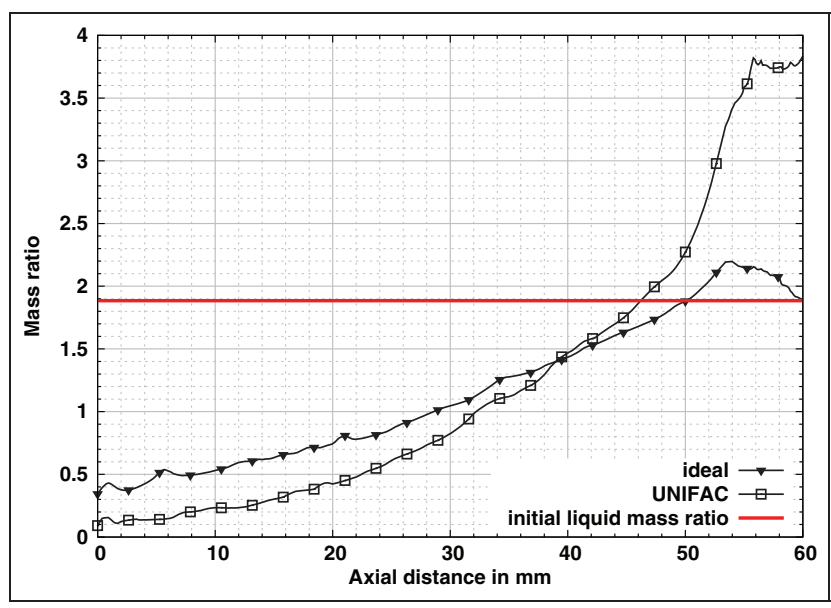

Figure 25. Vapor mass ratio of n-butanol to $n$-hexane along the spray axis for OP\#2 at I ms avSOI.

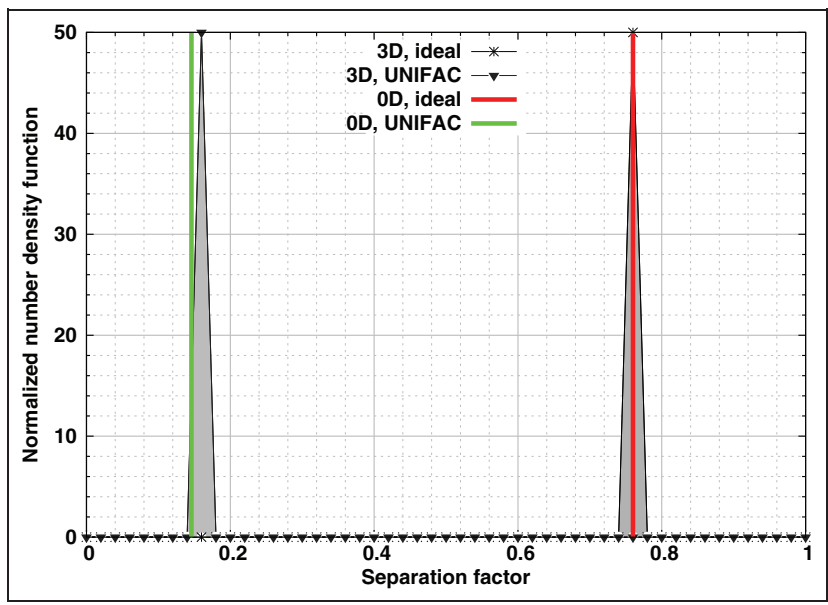

Figure 26. Separation factor of ethanol relative to $n$-hexane at an axial position of $30 \mathrm{~mm}$ for OP\#2 at I $\mathrm{ms}$ avSOI.

function is obtained by sampling over all droplets at an axial distance of $30 \mathrm{~mm}$. For the separation factors, the same values as for the single-droplet model are 


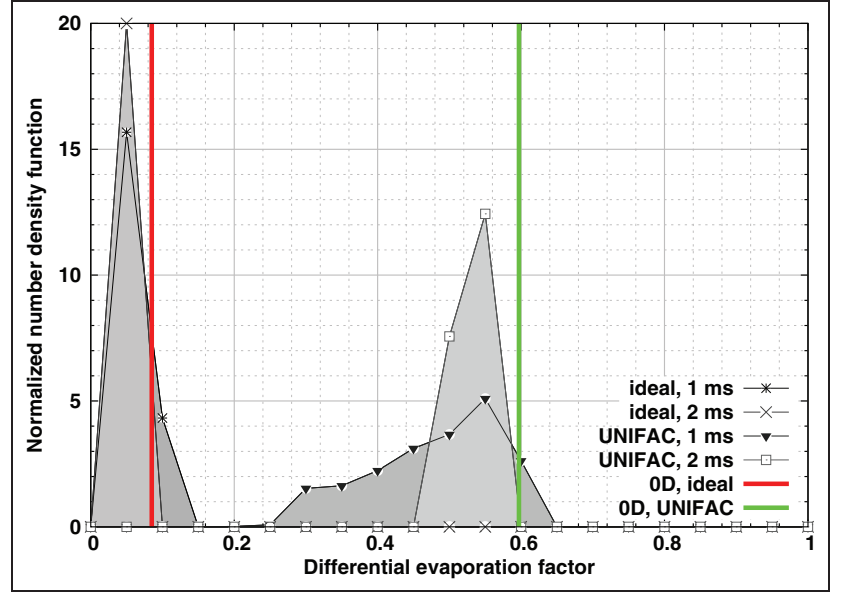

Figure 27. $D E F$ of ethanol relative to $n$-hexane at an axial position of $30 \mathrm{~mm}$ for OP\#2 at I ms and $2 \mathrm{~ms}$ avSOI.

obtained, where it is much lower with the UNIFAC method, and hence the evaporation of n-hexane dominates. The DEF also gives almost the same values as for the single-droplet model, where at $2 \mathrm{~ms}$ avSOI the $3 \mathrm{D}$ simulation results are even closer to those of the single-droplet simulation. The non-ideal results show a much stronger segregation of the Lagrangian droplets than the ideal model does. This again confirms the above findings for the vapor composition.

Based on these results, two important conclusions are found. First, it was discovered that for the EBH mixture considered, strong non-ideal effects occur which significantly affect the vapor composition. Second, the single-droplet model results can be directly transferred to the 3D CFD simulations with Lagrangian droplets. Hence, preliminary studies with the fast and easy-to-analyze $0 \mathrm{D}$ model can be used to characterize the vapor formation in combustion chambers in advance.

\section{Ternary mixture modification}

The EBH mixture investigated before was mainly composed of alcohols. Another realistic mixture representing the gasoline RON 95 is obtained by exchanging n-butanol with iso-octane using the same volumetric fraction. The resulting mixture ethanol/iso-octane/ $\mathrm{n}$-hexane $(\mathrm{EIH})$ is listed in Table 2. As illustrated in Keller et al., ${ }^{21}$ iso-octane and ethanol show a very interesting behavior regarding the sequence of evaporation as, depending on the position relative to the azeotropic point, either ethanol or iso-octane becomes the light end.

The VLE of the new binary mixtures are given in Figures 28 and 29. Now, non-ideal behavior is observed for the components with high mass fractions. On the other hand, the mixture of iso-octane and n-hexane behaves almost ideally.

The volume fractions of the EIH mixture are equal to those of the EBH mixture. The corresponding mole

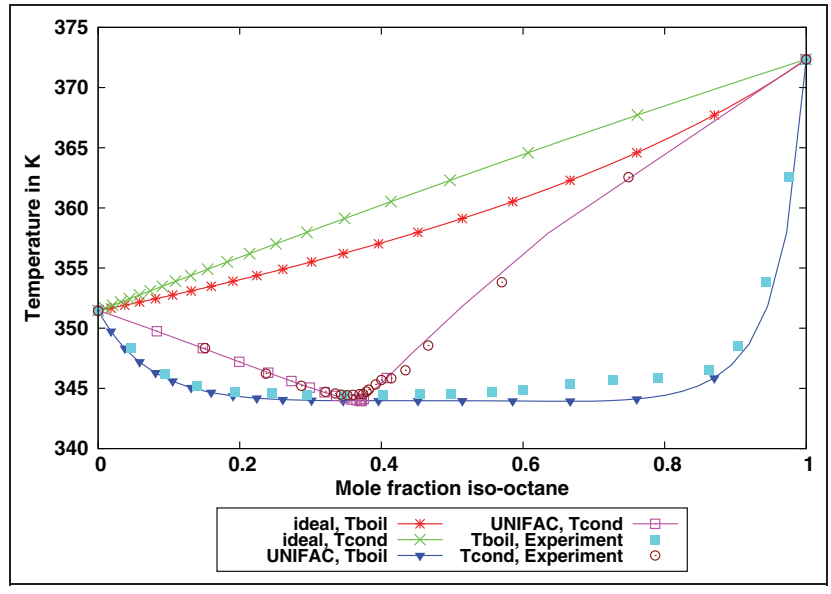

Figure 28. VLE of ethanol/iso-octane mixtures compared with experimental data from $\mathrm{Ku}$ and $\mathrm{Tu}^{29}$ at $\mathrm{p}=1.013$ bar.

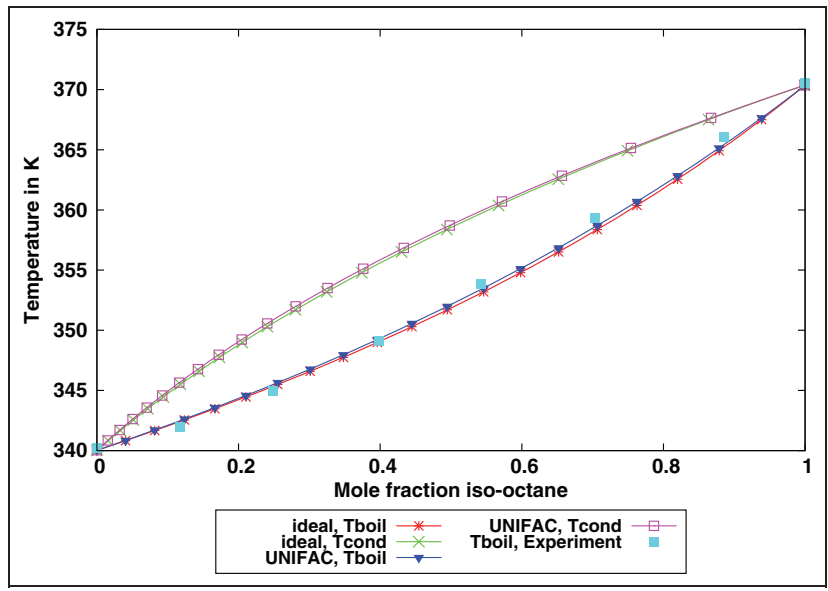

Figure 29. VLE of iso-octane/n-hexane mixtures compared with experimental data from Prasad et al. ${ }^{30}$ at $p=0.958$ bar.

fractions are now 88.5 mole \% ethanol, $6.3 \mathrm{~mole} \%$ iso-octane and $5.2 \mathrm{~mole} \% \mathrm{n}$-hexane. Again creating a binary mixture as a subset of the EIH mixture, while keeping the volume fraction ratios constant, the resulting binary mixture of ethanol and iso-octane would contain $93.4 \mathrm{~mole} \%$ ethanol and $6.6 \mathrm{~mole} \%$ isooctane, which is located to the left of the azeotropic point in Figure 28. In a phase diagram, the liquid always moves along the boiling temperature curve to the higher temperature. For the ideal behavior, this is pure iso-octane and for the non-ideal behavior it is ethanol. The masses of a droplet evaporated at OP\#2 reflect this accordingly, as illustrated in Figure 30. While, with the ideal method, iso-octane is contained until the end of DLT, it depletes with non-ideal behavior right after $\mathrm{n}$-hexane. The most volatile component n-hexane evaporates at a higher rate when the UNIFAC method is applied, while the evaporation behavior of ethanol is almost unchanged and only slightly slows down. The influence of the VLE model is also represented by the separation factor as given in 


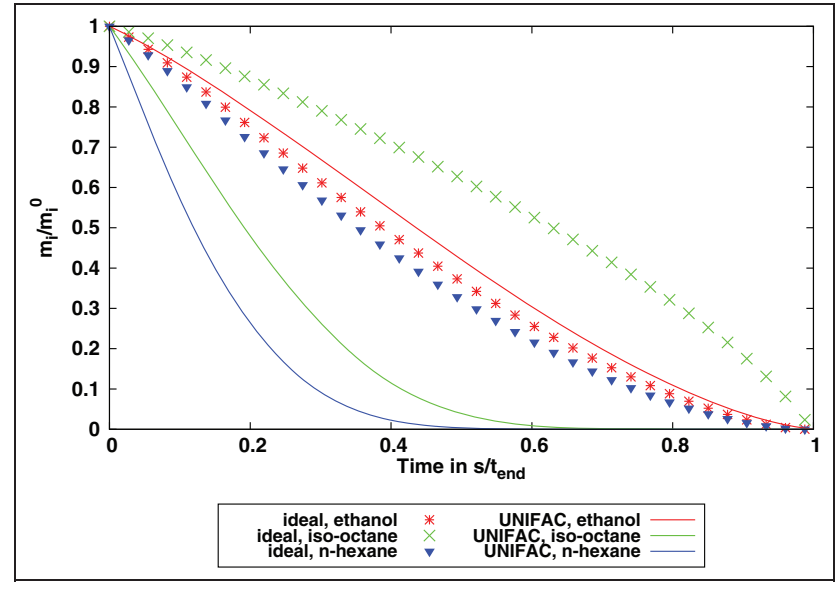

Figure 30. Temporal change in the masses of ElH mixture components at OP\#2.

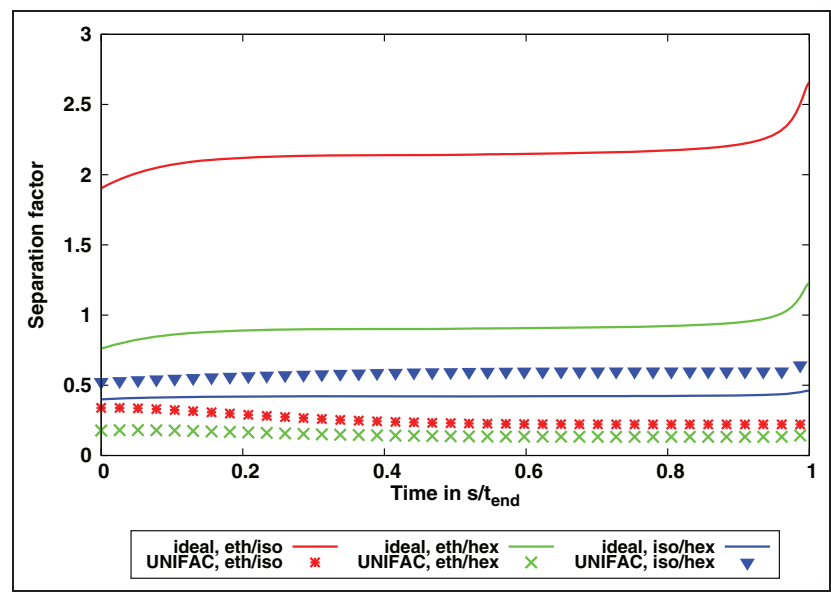

Figure 3I. Temporal evolution of separation factors of ElH mixture at OP\#2.
Figure 31. While the factors compared with n-hexane are always smaller than 1 , the ethanol/iso-octane values change for the different VLE methods. With ideal VLE, it is larger than 1, implying that ethanol leaves the droplet before iso-octane. However, with UNIFAC, it becomes smaller than 1 , which confirms that iso-octane has a higher evaporation rate.

As discussed before, the single-droplet investigation trends were also found in 3D CFD simulations. Next, 3D CFD simulation results are discussed to assess whether it is found again for this highly non-ideal mixture EIH. The vapor distribution obtained from the simulation is illustrated in Figure 32. The differences for ethanol and iso-octane are clearly visible. Obviously, the ethanol vapor is higher near the injector position in the left spray (ideal VLE), while iso-octane is much higher for the right spray (UNIFAC). Figure 33 shows both the vapor mass ratios and the initial liquid mass ratio of ethanol to iso-octane. While the ethanol mass is much higher than the iso-octane mass in the case of the ideal model, which is due to the depletion of ethanol in the droplets, it decreases to a value far below the ideal one with the non-ideal approach. Looking at the contour plots, it can be seen that although the ethanol evaporation rate decreases, there is still a large amount of ethanol vapor in the gas phase. Hence, the lower value of the mass ratio is caused by the depletion of iso-octane, which confirms the results obtained with the single-droplet model.

Finally, the separation factor of ethanol relative to iso-octane is given in Figure 34. The spray simulation results again reflect the single-droplet values very well, which gives the opportunity to use the single-droplet solver as a first step to evaluate the differential evaporation behavior in multicomponent sprays.

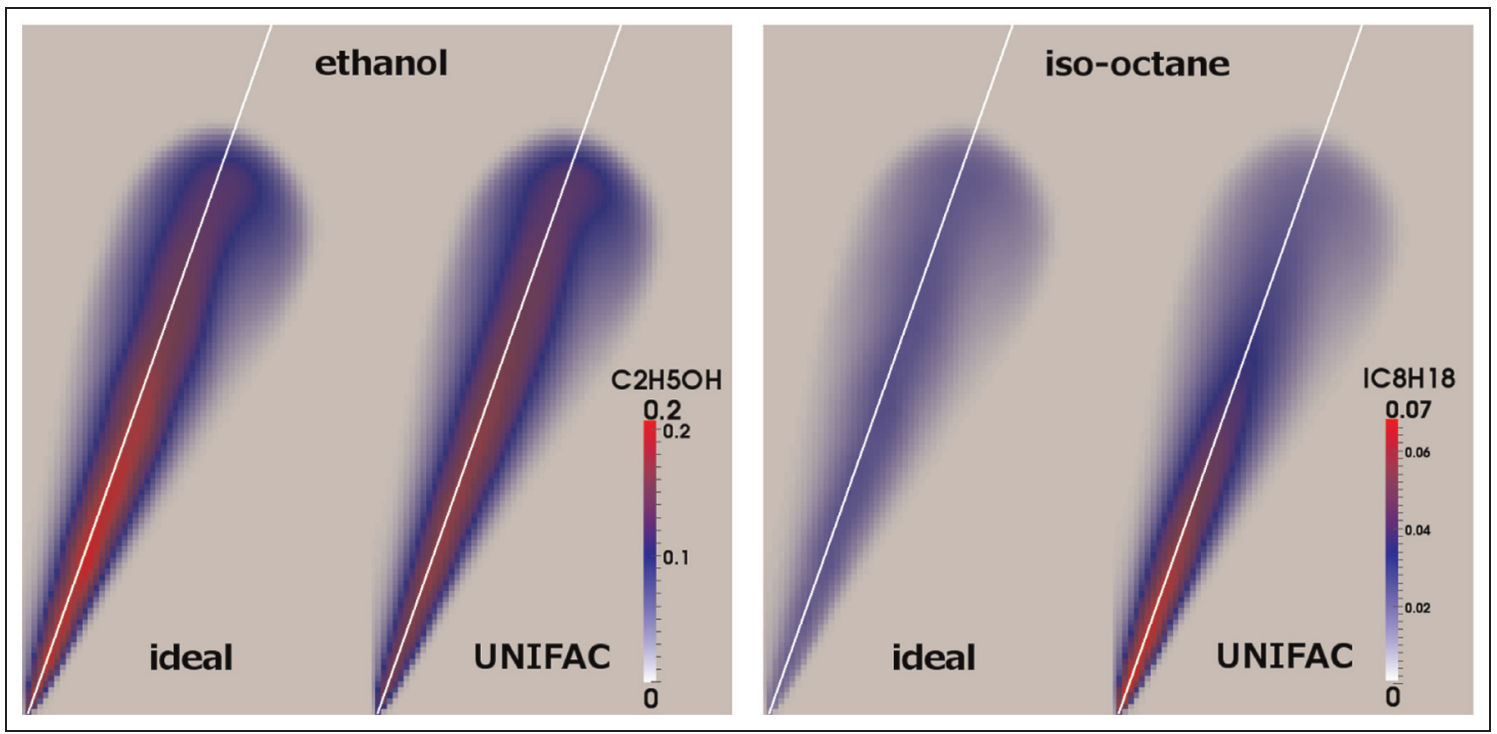

Figure 32. Contour plot of ethanol (left) and iso-octane (right) for the ElH mixture at OP\#2 simulated with ideal (left sprays) and UNIFAC method (right sprays) at I ms avSOI. 


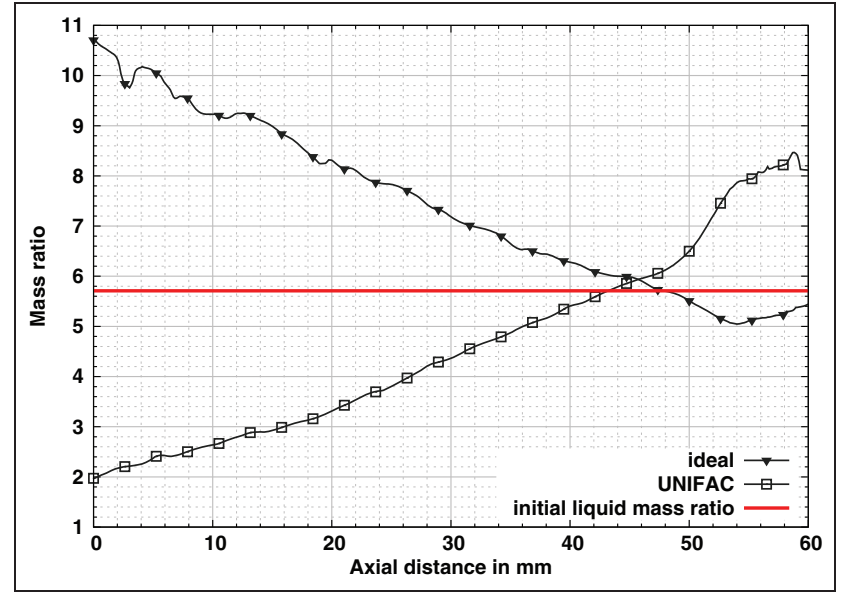

Figure 33. Vapor mass ratio of ethanol to iso-octane along the spray axis for OP\#2 at I ms avSOI.

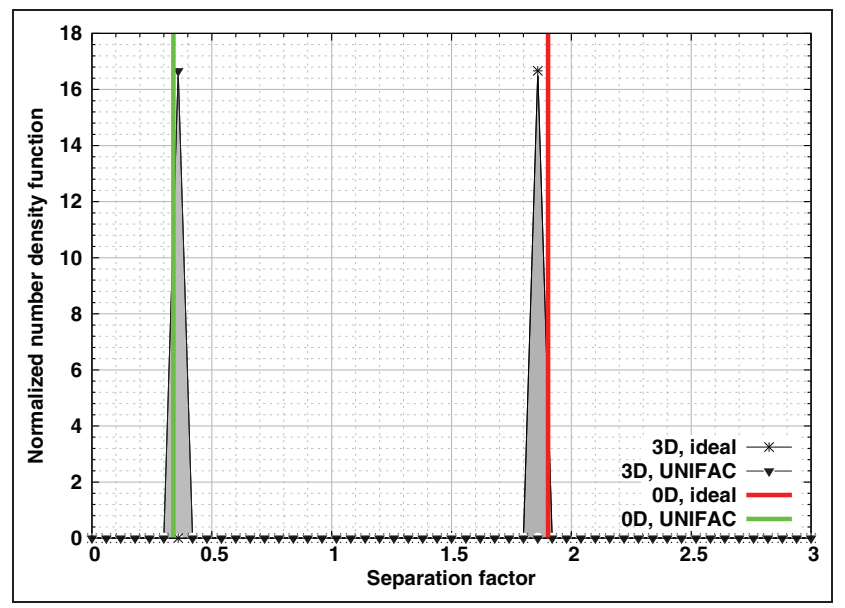

Figure 34. Separation factor of ethanol relative to iso-octane at an axial position of $30 \mathrm{~mm}$ for OP\#2 at I $\mathrm{ms}$ avSOI.

\section{Summary and conclusion}

The scope of this work is to investigate the differential evaporation behavior of a ternary EBH mixture. This is carried out with both single-droplet and full 3D CFD simulations, which are discussed and compared with the experimental data where available.

Looking at the binary VLE of each subset of the ternary mixture, it was found that the ethanol/n-butanol mixture behaves almost ideally because of the similar structure, while for the other two, ethanol/n-hexane and $\mathrm{n}$-butanol $/ \mathrm{n}$-hexane, an azeotrope is formed. Hence, the latter two mixtures show strong non-ideal behavior. Since UNIFAC yields better results than NRTL when compared with the available experimental data, it is used for the subsequent studies.

Looking at the single-droplet simulations, it can be observed that the DLTs and surface temperatures are almost unaffected when the ternary mixture is replaced with an ethanol/n-hexane mixture, but changes significantly for the other two binary mixtures. Furthermore, the choice of VLE model mainly affects the liquid composition and surface temperatures, but not the DLTs. The differential evaporation behavior and mixture separation is quantified with the DEF and separation factor, respectively. A modification is presented to account for an arbitrary number of components and the influence of the VLE model. For the selected mixtures, the differential evaporation is stronger if the non-ideal model (i.e. the UNIFAC method) is applied, while with ideal model the components evaporate almost simultaneously. Especially, mixtures composed of structurally dissimilar components such as the ethanol/n-hexane mixture, which have similar boiling temperatures and vapor pressures, show a strong dependency of the differential evaporation on the thermodynamic model.

The 3D CFD simulations are validated with the experimental data for the liquid (shadowgraphy results) and the vapor penetration depths (Schlieren). PDA measurements of the droplet sizes are used to define the droplet size distribution at the injection position.

The differential evaporation is demonstrated with contour plots showing significant differences for the two VLE approaches. Furthermore, the vapor mass ratios, separation factors and DEFs illustrate the comparability of the $0 \mathrm{D}$ single-droplet results with the full 3D CFD simulations. The same evaporation effects concerning the mixture separation as well as the results' dependency on the VLE model are obtained in the spray simulation. The effect of differential evaporation is increased by the real thermodynamic behavior, and this is quantified for both the average value and the distribution of the DEF.

The EBH mixture investigations demonstrate that the content of $10 \mathrm{vol} \% \mathrm{n}$-alkane in the mixture with alcohols causes major deviations from ideal behavior. As soon as $n$-hexane is completely evaporated, the remaining ethanol/n-butanol mixture evaporates separately but almost ideally.

Additionally, another mixture replacing n-butanol with iso-octane, the most widely used representative single-component gasoline surrogate, is investigated, showing strong non-ideal behavior. This is found again not only in the single-droplet studies but also in 3D CFD simulations. Significant differences in the mixture decomposition are found for the two VLE methods. While ethanol leaves the droplets before iso-octane with Raoult's law, with UNIFAC iso-octane depletes first. This change in the differential evaporation affects the vapor composition, as illustrated in the contour plots.

Finally, it is important to note that biofuel components such as alcohols lead to a considerable differential evaporation behavior. The resulting vapor fields differ strongly in their relative proportions to the initial liquid compositions. This is further increased by the non-ideal thermodynamic behavior. 


\section{Declaration of conflicting interests}

The authors declare that there is no conflict of interest.

\section{Funding}

The authors gratefully acknowledge the financial support by the Federal Ministry of Education and Research of Germany in the framework of Virtuhcon (project number 03Z2FN11), the Erlangen Graduate School in Advanced Technologies (SAOT), the Bavarian Research Foundation in the project WiDiKO_Wirkkette Direkteingespritzter Kraftstoffe im Ottomotor (project number AZ-932-10) and the German Research Foundation (DFG, INST 90/688-1).

\section{References}

1. Landis RB and Mills AF. Effect of internal diffusional resistance on the evaporation of binary droplets. In: Proceedings of the 5th international heat transfer conference, Tokyo, Japan, 3-7 September 1974, paper B7.9. Danbury, CT: Begell House Publishers.

2. Law CK. Internal boiling and superheating in vaporizing multicomponent droplets. AIChE J 1978; 24: 626-632.

3. Makino A and Law C. Heterogeneous flame propagation in the self-propagating high-temperature synthesis (SHS) process: Theory and experimental comparisons. In: 24th international symposium on combustion, proceedings of the Combustion Institute, University of Sydney, Sydney, Australia, 5-10 July 1992, pp.1883-1891. Amsterdam, Netherlands: Elsevier.

4. Kneer R, Schneider M, Noll B and Wittig S. Diffusion controlled evaporation of a multicomponent droplet: theoretical studies on the importance of variable liquid properties. Int J Heat Mass Tran 1993; 36: 2403-2415.

5. Sirignano WA. Fluid dynamics and transport of droplets and sprays. 2nd ed. Cambridge: Cambridge University Press, 2010.

6. Keller P, Bader A and Hasse C. The influence of intradroplet heat and mass transfer limitations in evaporation of binary hydrocarbon mixtures. Int $J$ Heat Mass Tran 2013; 67: 1191-1207.

7. Abramzon B and Sirignano W. Droplet vaporization model for spray combustion calculations. Int $J$ Heat Mass Tran 1989; 32: 1605-1618.

8. Wilms J. Evaporation of multicomponent droplets. PhD Thesis, Universität Stuttgart, Stuttgart, 2005.

9. Bader A, Keller P and Hasse C. The influence of nonideal vapor-liquid equilibrium on the evaporation of ethanol/iso-octane droplets. Int J Heat Mass Tran 2013; 64: 547-558.

10. Turns SR. An introduction to combustion: concepts and applications. 2nd ed. New York: McGraw-Hill, 2000.

11. Sazhin SS. Advanced models of fuel droplet heating and evaporation. Prog Energ Combust 2006; 32: 162-214.

12. Law CK. Multicomponent droplet combustion with rapid internal mixing. Combust Flame 1976; 26: 219-233.

13. Sirignano WA and Law CK. Transient heating and liquid-phase mass diffusion in droplet vaporization. In: Zung JT (ed.) Evaporation combustion of fuels (Advances in chemistry series, vol. 166). Washington, DC: American Chemical Society, 1978, pp.3-26.
14. Kralj C. Numerical simulation of diesel spray processes. $\mathrm{PhD}$ Thesis, Imperial College London, London, 1996.

15. Nordin N. Complex chemistry modeling of diesel spray combustion. PhD Thesis, Chalmers University of Technology, Gothenburg, 2001.

16. Launder BE and Sharma BI. Application of the energydissipation model of turbulence to the calculation of flow near a spinning disc. Lett Heat Mass Trans 1974; 1: 131-137.

17. Kärrholm F. Numerical modelling of diesel spray injection, turbulence interaction and combustion. $\mathrm{PhD}$ Thesis, Chalmers University of Technology, Gothenburg, 2008.

18. Wagner W. New vapour pressure measurements for argon and nitrogen and a new method for establishing rational vapour pressure equations. Cryogenics 1973; 13: 470-482.

19. Renon H and Prausnitz JM. Local compositions in thermodynamic excess functions for liquid mixtures. AIChE J 1968; 14: 135-144.

20. Gmehling J, Kolbe B, Kleiber M and Rarey J. Chemical thermodynamics for process simulation. Weinheim: Wiley$\mathrm{VCH}, 2012$.

21. Keller P, Knorsch T, Wensing M and Hasse C. Experimental and numerical analysis of iso-octane/ethanol sprays under gasoline engine conditions. Int $J$ Heat Mass Tran 2015; 84: 497-510.

22. Knorsch T, Zigan J, Wensing M and Leipertz A. Quantitative DISI spray vapor temperature study for different biofuels by two-line excitation laser-induced fluorescence. SAE paper 2012-01-1658, 2012.

23. Knorsch T, Heldmann M, Zigan L, Wensing M and Leipertz A. On the role of physiochemical properties for DISI biofuel spray evaporation. Exp Fluid 2013; 54: $1-14$.

24. Lucchini T, D'Errico G and Ettorre D. Numerical investigation of the spray-mesh-turbulence interactions for high-pressure, evaporating sprays at engine conditions. Int $J$ Heat Fluid Fl 2011; 32: 285-297.

25. Seo MD, Kim YJ, Lim JS and Kang JW. Measurement and correlation of the isobaric vapor-liquid equilibrium for mixtures of alcohol + ketone systems at atmospheric pressure. Korean J Chem Eng 2012; 29: 103-110.

26. Kudryavtseva L and Susarev MMP. Liquid-vapor equilibrium in the system of acetone-hexane and hexane-ethyl alcohol at 35, 45 and 55 and $760 \mathrm{~mm}$ of $\mathrm{Hg}$. J Appl Chem 1963; USSR 26: 1419-1424.

27. Dominguez M, Mainar A, Artigas H, Royo F and Urieta J. Isobaric VLE data of the ternary system (1-Butanol + $n$-Hexane +1 -Butylamine) and the three constituent binary mixtures at $101.3 \mathrm{kPa}$. J Chem Eng Jpn 1997; 30: $484-490$.

28. Xiao F, Dianat M and McGuirk J. LES of turbulent liquid jet primary breakup in turbulent coaxial air flow. Int J Multiphas Flow 2014; 60: 103-118.

29. $\mathrm{Ku} \mathrm{H}-\mathrm{C}$ and $\mathrm{Tu} \mathrm{C}-\mathrm{H}$. Vapor-liquid equilibria for binary and ternary mixtures of diisopropyl ether, ethanol, and 2,2,4-trimethylpentane at $101.3 \mathrm{kPa}$. Fluid Phase Equilibr 2006; 248: 197-205.

30. Prasad TV, Sriram N, Raju A and Prasad D. (Vapor + liquid) equilibria of binary mixtures formed by iso-octane with a variety of compounds at $95.8 \mathrm{kPa}$. J Chem Thermodyn 2006; 38: 119-122.

31. Watson, KM. Thermodynamics of the liquid state. Ind Eng Chem 1943; 35: 398-406. 


\section{Appendix I}

\section{Heat of vaporization}

The heat of vaporization determined with the Watson equation $^{31}$ is given for the components EBH in Figure 35.

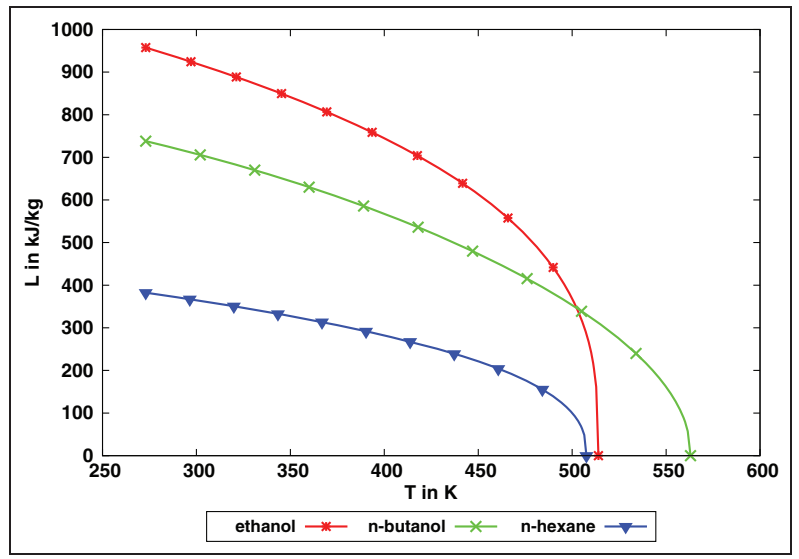

Figure 35. Heat of vaporization of ethanol, n-butanol and n-hexane.

\section{Appendix 2}

\section{Vapor pressure}

The vapor pressures calculated with the Wagner equation given in Wagner ${ }^{18}$ are illustrated for the components EBH in Figure 36.

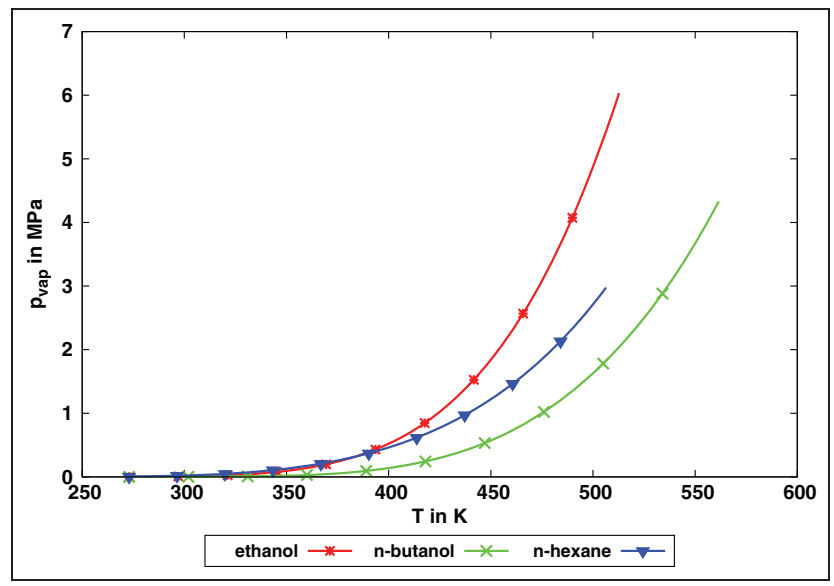

Figure 36. Vapor pressures of ethanol, n-butanol and n-hexane. 